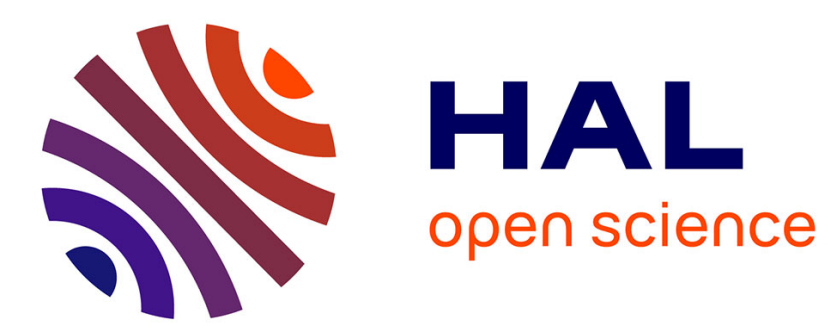

\title{
ASYMPTOTIC BEHAVIOUR OF CURVED RODS BY THE UNFOLDING METHOD \\ Georges Griso
}

\section{To cite this version:}

Georges Griso. ASYMPTOTIC BEHAVIOUR OF CURVED RODS BY THE UNFOLDING METHOD. Mathematical Methods in the Applied Sciences, 2004, 27 (17), pp.2081-2110. 10.1002/mma.546 . hal-00620202

\section{HAL Id: hal-00620202 https://hal.science/hal-00620202}

Submitted on 9 Sep 2011

HAL is a multi-disciplinary open access archive for the deposit and dissemination of scientific research documents, whether they are published or not. The documents may come from teaching and research institutions in France or abroad, or from public or private research centers.
L'archive ouverte pluridisciplinaire HAL, est destinée au dépôt et à la diffusion de documents scientifiques de niveau recherche, publiés ou non, émanant des établissements d'enseignement et de recherche français ou étrangers, des laboratoires publics ou privés. 


\title{
ASYMPTOTIC BEHAVIOUR OF CURVED RODS BY THE UNFOLDING METHOD
}

\author{
G. GRISO \\ Laboratoire J.-L. Lions-CNRS, Boîte courrier 187, Université Pierre et Marie Curie, \\ 4 place Jussieu, 75005 Paris, France, Email: griso@ann.jussieu.fr
}

\begin{abstract}
We consider in this work general curved rods with a circular cross-section of radius $\delta$. Our aim is to study the asymptotic behaviour of such rods as $\delta \rightarrow 0$, in the framework of the linear elasticity according to the unfolding method. It consists in giving some decompositions of the displacements of such rods, and then in passing to the limit in a fixed domain.

A first decomposition concerns the elementary displacements of a curved rod which characterize its translations and rotations, and the residual displacements related to the deformation of the cross-section. The second decomposition concerns the displacements of the middle-line of the rod. We prove that such a displacement can be written as the sum of an inextensional displacement and of an extensional one. An extensional displacement will modify the length of the middle-line, while an inextensional displacement will not change this length in a first approximation. We show that the $H^{1}$-norm of an inextensional displacement is of order 1 , while that of an extensional displacement is in general, of order $\delta$.

A priori estimates are established and convergence results as $\delta \rightarrow 0$, are given for the displacements. We give their unfolded limits, as well as the unfolded limits of the strain and stress tensors. To prove the convergence of the strain tensor, the introduction of elementary and residual displacements appears as essential. By passing to the limit as $\delta \rightarrow 0$ in the linearized system of the elasticity, we obtain on the one hand, a variational problem that is satisfied by the limit extensional displacement, and on the other hand, a variational problem coupling the limit of inextensional displacements and the limit of the angle of torsion.
\end{abstract}

\section{Introduction}

The rods we consider here are thin curved cylinders built around a middle-line which is a smooth curve in $\mathbb{R}^{3}$. To be more precise, given a curve $\zeta$ in $\mathbb{R}^{3}$, the cross-section of a rod with the middle-line $\zeta$ (i.e., its intersection with a normal plane at each point $M$ of $\zeta$ ), is a disk $D(M ; \delta)$ of radius $\delta$.

Our work is essentially based on two decompositions of the displacements of the rod. Under the action of any displacement $u^{\delta}$, the disk $D(M ; \delta)$ is submitted on the one hand to a translation and a rotation, that we call elementary displacements of the rod, and, on the other hand, to a displacement that deforms the disk, called residual displacement.

The second decomposition concerns the displacements of the middle-line of the rod. We prove that such a displacement can be written as the sum of an inextensional displacement and of an extensional one. As suggested by its name, an extensional displacement will modify the length of the middle-line, while an inextensional displacement will not change this length in a first approximation. If the $H^{1}$-norm of the inextensional displacement is of order 1 , that of the extensional displacement is in general, of order $\delta$.

The proofs of a priori estimates are based on the method used by Kondratiev and Oleinik [9] and by Cioranescu, Oleinik and Tronel [2] to establish Korn inequalities for frame-type structures and junctions.

Once a priori estimates are established, we study the convergence, as $\delta \rightarrow 0$, of the displacements of a curved rod. We give their unfolded limits, as well as the unfolded limits of the strain and stress tensors.

In the next step, we turn our attention to the linearized system of the elasticity written for a family of curved rods. By passing to the limit as $\delta \rightarrow 0$, we obtain on the one hand, a variational problem that is satisfied by the limit extensional displacement, and on the other hand, a variational problem coupling the 
limit of inextensional displacement and the limit of the angle of torsion, see Theorems 7.1 and 7.2 below. To establish these results, we first prove the convergence of the strain tensor. As can be seen in the sequel, this is the step where the introduction of elementary and residual displacements appears as essential.

There is a vast litterature on the asymptotic models for straight rods, e.g., Griso [4], Le Dret [10], Murat and Sili [12], [13], Trabucho and Viano [18]. Concerning previous works on curved rods, let us mention that of Arunakinirathar and Reddy [1] whose aim is to present a mechanical theory in this field. The first mathematical approaches of the asymptotic behaviour of curved rods is due to Jamal and SanchezPalencia [6], Sanchez-Hubert and Sanchez-Palencia [15], Sanchez-Palencia [16], and Jurak and Tambača [7], [8], Tambača [17]. In these papers, by using thin domains techniques and multiple-scale methods, the authors obtain a limit problem coupling the inextensional displacement and the limit of the angle of torsion. In [15] and [16] an interpretation of the limit stress tensor is also given. Let us mention also the work of Neunkirch and al [14] where very interesting numerical results are presented.

The paper is organized as follows: in Section 1 we introduce the notation, make preliminary hypotheses, recall some results that will be used in the sequel and give the definition of a curved rod. We also recall the definition of the unfolding operator for thin rods. The same operator for the case of plates was introduced in Griso [5]; for the periodic case, we refer the reader to Cioranescu, Damlamian and Griso [3]. In Section 2 we show that for star-shaped domains, one can always approach an $H^{1}$ - displacement by a rigid body one. In Section 3 we define the class of elementary displacements associated to a curved rod. Theorem 3.3 gives sharp estimates with respect to $\delta$ for this kind of displacements. Section 4 defines the notions of elementary displacements and residual displacements, and introduces the two decompositions mentioned above. The first decomposition is based on elementary displacements and the second one uses the residual ones. In Section 5 we suppose that we are given a bounded sequence of displacements and we study the limit as $\delta \rightarrow 0$ of a convergent subsequence. We also give the limit of the unfolded strain tensor. In Section 6 , the linearized problem of elasticity for a curved rod is formulated. We show that under appropriate hypotheses on the applied forces, the solutions (i.e., the displacements of the rod) are bounded, so that the convergence results from Section 5 can be used. Further results, concerning the convergence of the stress tensor are also established. Finally, in Section 7 the complete characterization of the limit problem is given. The last section is dedicated to comments and remarks.

\section{Notations and preliminaries}

Let $\zeta$ be a smooth curve belonging to the euclidian space $\mathbb{R}^{3}$. This curve is parametrized as follows

$$
\overrightarrow{O M}(s)=\phi(s),
$$

where $s$ is the arc length and $\phi$ is a function belonging to $\mathcal{C}^{3}\left(0, L ; \mathbb{R}^{3}\right)$.

Introduce the Frenet frame

$$
\begin{cases}\frac{d \overrightarrow{O M}}{d s}=\vec{T}, & \|\vec{T}\|_{\mathbb{R}^{3}}=1 \\ \frac{d \vec{T}}{d s}=c \vec{N}, & \|\vec{N}\|_{\mathbb{R}^{3}}=1 \\ \vec{B}=\vec{T} \wedge \vec{N}, & \end{cases}
$$

where $\|\cdot\|_{\mathbb{R}^{3}}$ is the euclidian norm in $\mathbb{R}^{3}$.

We have

$$
\left\{\begin{array}{l}
\frac{d \vec{N}}{d s}=-c \vec{T}+\tau \vec{B}, \\
\frac{d \vec{B}}{d s}=-\tau \vec{N}
\end{array}\right.
$$


where $c=c(s)$ is the curvature and $\tau=\tau(s)$ is the torsion of the curve $\zeta$ in the point $M(s)$.

In the sequel, $D(a ; r)$ denotes the open disc of center $a$ and radius $r$ (while obviously, $\bar{D}(a ; r)$ denotes the closed disc).

In this work we assume that the following hypothesis holds true:

Нypothesis 1.1. The Frenet frame $(M ; \vec{T}, \vec{N}, \vec{B})$ given by (1.1) and (1.2), is defined in any point of the interval $[0, L]$ and belongs to $\mathcal{C}^{1}\left(0, L ;\left(\mathbb{R}^{3}\right)^{4}\right)$. Moreover, the function $\phi$ is one-to-one.

For more details, we refer the reader to [11].

Introduce now the mapping $\Phi:[0, L] \times \mathbb{R}^{2} \longrightarrow \mathbb{R}^{3}$ defined by

$$
\Phi \quad: \quad\left(s, s_{2}, s_{3}\right) \longmapsto \overrightarrow{O M}(s)+s_{2} \vec{N}(s)+s_{3} \vec{B}(s)
$$

There exists $\delta_{0} \in \mathbb{R}$ depending only on $\zeta$, such that the restriction of $\Phi$ to the compact set $[0, L] \times \bar{D}\left(O ; \delta_{0}\right)$ is a $\mathcal{C}^{1}-$ diffeomorphism of that set onto its range. As a matter of fact $\delta_{0}$ is such that

$$
0<\delta_{0} \leq \frac{1}{2\|c\|_{L^{\infty}(0, L)}}
$$

Definition 1.2. The curved rod $\mathcal{P}_{\delta}$ is defined as follows:

$$
\left.\left.\mathcal{P}_{\delta}=\Phi(] 0, L[\times D(O ; \delta)), \quad \text { for } \delta \in\right] 0, \delta_{0}\right] .
$$

A portion of $\mathcal{P}_{\delta}$ is defined by

$$
\mathcal{P}_{\delta}\left(t, t^{\prime}\right)=\Phi(] t, t^{\prime}[\times D(O ; \delta)), \quad 0 \leq t<t^{\prime} \leq L .
$$

The boundary of the rod $\mathcal{P}_{\delta}$ is made, on one hand, of the two discs $D(M(0) ; \delta)$ and $D(M(L) ; \delta)$, belonging to the normal plane to the curve $\zeta$ in the points $M(0)$ and $M(L)$, respectively. On the other hand, the boundary of $\mathcal{P}_{\delta}$ also contains the lateral surface of the rod. This surface is parametrized by $(s, \theta) \longmapsto K(s, \theta)$, where

$$
K(s, \theta)=\overrightarrow{O M}(s)+\delta(\vec{N}(s) \cos \theta+\vec{B}(s) \sin \theta), \quad s \in[0, L], \quad \theta \in[0,2 \pi]
$$

Notation 1.3. (i). Reference domains and running points. We denote by $x$ the running point of $\mathcal{P}_{\delta}$, by $d x$ the volume element and by $d_{s} x$ the surface one in $\mathcal{P}_{\delta}$. The running point of the cylinder $\left.\omega_{\delta}=\right] 0, L[\times D(O ; \delta)$ is $\left(s, s_{2}, s_{3}\right)$. To simplify, $d \omega_{\delta}$ denotes the volume element $d s d s_{2} d s_{3}$ in $\omega_{\delta}$, while $d_{s} \omega_{\delta}$ denotes the surface one. The reference domain

$$
\omega=] 0, L[\times D(O ; 1),
$$

is obtained by transforming $\omega_{\delta}$ by an orthogonal affinity of ratio $1 / \delta$. The running point of $\omega$ is $\left(s, S_{2}, S_{3}\right)$, $d \omega$ and $d_{s} \omega$ denote respectively, the volume element and the surface element in $\omega$.

(ii). Displacements. For any displacement $u \in H^{1}\left(\mathcal{P}_{\delta} ; \mathbb{R}^{3}\right)$, we write $u$ instead of $u \circ \Phi$.

The Unfolding Operator. Let $w$ be a function defined on $\omega_{\delta}$. We denote $\mathcal{T}_{\delta}(w)$ the function defined on $\omega$ as follows:

$$
\mathcal{T}_{\delta}(w)\left(s, S_{2}, S_{3}\right)=w\left(s, \delta S_{2}, \delta S_{3}\right), \quad \forall\left(s, S_{2}, S_{3}\right) \in \omega .
$$


REMARK 1.4. Obviously, if $w \in L^{2}\left(\omega_{\delta} ; \mathbb{R}^{k}\right)$, then $\mathcal{T}_{\delta}(w) \in L^{2}\left(\omega ; \mathbb{R}^{k}\right)$.

When not precised, $C$ will denote in the whole work, different constants independent of $\Omega, \omega$ and $\delta$. For any function $\psi \in L^{2}(\Omega), \mathcal{M}_{\Omega}(\psi)$ denotes the mean value of $\psi$ in $\Omega$,

$$
\mathcal{M}_{\Omega}(\psi)=\frac{1}{\text { measure } \Omega} \int_{\Omega} \psi(x) d x
$$

We will make use several times of the Poincaré-Wirtinger inequality in star-shaped domains with respect to a ball. For the reader's convenience we give here its proof.

Proposition 1.5. ( Poincaré-Wirtinger Inequality). Let $\Omega$ be a bounded open set in $\mathbb{R}^{n}$ of diameter $R$. Assume that $\Omega$ is star-shaped with respect to the ball $B\left(O ; R_{1}\right)$. Then, for all $\phi \in H_{l o c}^{1}(\Omega)$,

$$
\left\{\begin{array}{l}
\left\|\phi-\mathcal{M}_{\Omega}(\phi)\right\|_{L^{2}(\Omega)}^{2} \leq C\left(\frac{R}{R_{1}}\right)^{n+1} \int_{\Omega} \rho^{2}(x)|\nabla \phi(x)|^{2} d x, \\
\left\|\phi-\mathcal{M}_{B\left(O ; R_{1}\right)}(\phi)\right\|_{L^{2}(\Omega)}^{2} \leq C\left(\frac{R}{R_{1}}\right)^{n+1} \int_{\Omega} \rho^{2}(x)|\nabla \phi(x)|^{2} d x
\end{array}\right.
$$

where the constant $C$ depends only on $n$ and $\rho(x)$ is the distance from $x$ to the boundary $\partial \Omega$.

The proof of Proposition 1.5 is based on the following result:

Lemma 1.6. Let $\Omega$ be a bounded open set in $\mathbb{R}^{n}$ of diameter $R$. Assume that $\Omega$ is star-shaped with respect the ball $B\left(O ; R_{1}\right)$. Then

$$
\|\phi\|_{L^{2}(\Omega)}^{2} \leq\left(2^{n+5}+1\right) \frac{R^{n+1}}{R_{1}^{n+1}}\left[\|\phi\|_{L^{2}\left(B\left(O ; R_{1}\right)\right.}^{2}+\int_{\Omega} \rho^{2}(x)|\nabla \phi(x)|^{2} d x\right], \quad \forall \phi \in H_{l o c}^{1}(\Omega) .
$$

Proof of the lemma 1.6. As said above, the proof is based on arguments used by Kondratiev and Oleinik [9] (see also [2]). Let $\theta$ be a function in $\mathcal{C}^{\infty}\left(\mathbb{R}^{+}\right)$such that

$$
\begin{cases}\theta(t)=0 & \text { if } 0 \leq t \leq \frac{R_{1}}{2} \\ \theta(t)=1 & \text { if } t \geq R_{1}, \\ 0 \leq \theta(t) \leq 1 & \text { if } \frac{R_{1}}{2} \leq t \leq R_{1} \\ \left|\theta^{\prime}(t)\right| \leq \frac{4}{R_{1}}, & \forall t \in \mathbb{R}^{+} .\end{cases}
$$

Let also $\phi \in \mathcal{C}^{\infty}\left(\mathbb{R}^{n}\right)$, and consider the ray (segment) $(O, P)$ joining the origin $O$ to an arbitrary point $P \in \partial \Omega$. We denote by $\ell$ its length, $\ell=\|O P\|_{\mathbb{R}^{3}}$. The Hardy inequality yields,

$$
\int_{0}^{\ell}|\theta(r) \phi(x)|^{2} d r \leq 4 \int_{0}^{\ell}|r-\ell|^{2}\left|\frac{\partial(\theta \phi)}{\partial r}\right|^{2} d r
$$

where $r=\|O x\|_{\mathbb{R}^{3}}$ with $x \in(O, P)$. Since

$$
|r-\ell|=\operatorname{dist}(x, P) \leq \frac{R}{R_{1}} \rho(x) \quad \text { and } \quad|r-\ell| \leq R,
$$


from the properties of $\theta$ and (1.8) one has

$$
\begin{aligned}
\frac{1}{R^{n-1}} & \int_{R_{1}}^{\ell}|\phi(x)|^{2} r^{n-1} d r \leq \int_{R_{1}}^{\ell}|\phi(x)|^{2} d r \\
& \leq 4\left(\frac{R}{R_{1}}\right)^{2}\left[\int_{\frac{R_{1}}{2}}^{\ell} \rho^{2}(x)|\nabla \phi(x)|^{2} d r+16 \int_{\frac{R_{1}}{2}}^{R_{1}}|\phi(x)|^{2} d r\right] \\
& \leq 4 \frac{R^{2}}{R_{1}^{2}} \frac{2^{n-1}}{R_{1}^{n-1}}\left[\int_{\frac{R_{1}}{2}}^{\ell} \rho^{2}(x)|\nabla \phi(x)|^{2} r^{n-1} d r+16 \int_{\frac{R_{1}}{2}}^{R_{1}}|\phi(x)|^{2} r^{n-1} d r\right] .
\end{aligned}
$$

Multiplying (1.9) by $\left(\frac{R_{1}}{2}\right)^{n-1}$, and integrating on all the rays, leads to

$$
\int_{\Omega \backslash B\left(O ; R_{1}\right)}|\phi(x)|^{2} d x \leq 2^{n+5} \frac{R^{n+1}}{R_{1}^{n+1}}\left[\int_{\Omega} \rho(x)^{2}|\nabla \phi(x)|^{2} d x+\int_{B\left(O ; R_{1}\right)}|\phi(x)|^{2} d x\right],
$$

whence

$$
\|\phi\|_{L^{2}(\Omega)}^{2} \leq\left(2^{n+5}+1\right) \frac{R^{n+1}}{R_{1}^{n+1}}\left[\int_{\Omega} \rho(x)^{2}|\nabla \phi(x)|^{2} d x+\int_{B\left(O ; R_{1}\right)}|\phi(x)|^{2} d x\right] .
$$

By density, inequality (1.10) is satisfied for all $\phi \in H^{1}(\Omega)$.

Now, choose $\phi \in H_{l o c}^{1}(\Omega)$ such that

$$
\int_{\Omega} \rho^{2}(x)|\nabla \phi(x)|^{2} d x<+\infty
$$

Denote by $\Omega_{\varepsilon}$ the image of $\Omega$ by the homothety of center $O$ and ratio $1-\varepsilon, 0<\varepsilon<1$. Since, obviously $\phi \in H^{1}\left(\Omega_{\varepsilon}\right)$, from (1.10) one has

$$
\begin{aligned}
\|\phi\|_{L^{2}\left(\Omega_{\varepsilon}\right)}^{2} & \leq\left(2^{n+5}+1\right) \frac{R^{n+1}}{R_{1}^{n+1}}\left[\int_{\Omega_{\varepsilon}} \rho_{\varepsilon}^{2}(x)|\nabla \phi(x)|^{2} d x+\int_{B\left(O ;(1-\varepsilon) R_{1}\right)}|\phi(x)|^{2} d x\right] \\
& \leq\left(2^{n+5}+1\right) \frac{R^{n+1}}{R_{1}^{n+1}}\left[\int_{\Omega} \rho^{2}(x)|\nabla \phi(x)|^{2} d x+\int_{B\left(O ; R_{1}\right)}|\phi(x)|^{2} d x\right],
\end{aligned}
$$

where $\rho_{\varepsilon}(x)$ is the distance from $x$ to $\partial \Omega_{\varepsilon}, \rho_{\varepsilon}(x) \leq \rho(x)$. Then, the result follows by passing to the limit as $\varepsilon \rightarrow 0$.

Proof of Proposition 1.5. Let $\phi \in H_{l o c}^{1}(\Omega)$. Applying the classical Poincaré-Wirtinger inequality to the restriction of $\phi$ to the ball $B\left(O ; R_{1} / 2\right)$, gives

$$
\left\|\phi-\mathcal{M}_{B\left(O ; R_{1} / 2\right)}(\phi)\right\|_{L^{2}\left(B\left(O ; R_{1} / 2\right)\right)}^{2} \leq C R_{1}^{2} \int_{B\left(O ; R_{1} / 2\right)}|\nabla \phi(x)|^{2} d x
$$

where $C$ depends only on $n$. One has successively,

$$
\begin{aligned}
\left\|\phi-\mathcal{M}_{B\left(O ; R_{1} / 2\right)}(\phi)\right\|_{L^{2}\left(B\left(O ; R_{1} / 2\right)\right)}^{2} & \leq C \int_{B\left(O ; R_{1} / 2\right)} \rho(x)^{2}|\nabla \phi(x)|^{2} d x \\
& \leq C \int_{\Omega} \rho^{2}(x)|\nabla \phi(x)|^{2} d x
\end{aligned}
$$


Now, let us apply Lemma 1.6 to the function $\phi-\mathcal{M}_{B\left(O ; R_{1} / 2\right)}(\phi)$ since $\Omega$ is obviously star-shaped with respect to the ball $B\left(O, R_{1} / 2\right)$. We get

$$
\left\|\phi-\mathcal{M}_{B\left(O ; R_{1} / 2\right)}(\phi)\right\|_{L^{2}(\Omega)}^{2} \leq C\left(\frac{R}{R_{1}}\right)^{n+1} \int_{\Omega} \rho^{2}(x)|\nabla \phi(x)|^{2} d x .
$$

On the other hand, by using Cauchy-Schwarz inequality, one has

$$
\left|\int_{\Omega} \phi d x-\int_{\Omega} \mathcal{M}_{B\left(O ; R_{1} / 2\right)}(\phi) d x\right|^{2} \leq\left(\int_{\Omega} d x\right)\left(\int_{\Omega}\left|\phi-\mathcal{M}_{B\left(O ; R_{1} / 2\right)}(\phi)\right|^{2} d x\right),
$$

so that, due to (1.12),

$$
\left|\mathcal{M}_{\Omega}(\phi)-\mathcal{M}_{B\left(O ; R_{1} / 2\right)}(\phi)\right|^{2} \leq \frac{C}{\text { measure } \Omega}\left(\frac{R}{R_{1}}\right)^{n+1} \int_{\Omega} \rho^{2}(x)|\nabla \phi(x)|^{2} d x,
$$

which, combined with (1.11) leads to the first inequality in (1.7).

Observe now that for any $x \in B\left(O ; R_{1}\right)$, one has

$$
\left|R_{1}-\|O x\|_{\mathbb{R}^{3}}\right| \leq \rho(x) .
$$

Therefore, applying the first inequality in (1.7) to the restriction of $\phi$ to the ball $B\left(O ; R_{1}\right)$, one gets

$$
\left\|\phi-\mathcal{M}_{B\left(O ; R_{1}\right)}(\phi)\right\|_{L^{2}\left(B\left(O ; R_{1}\right)\right)}^{2} \leq C \int_{\Omega} \rho^{2}(x)|\nabla \phi(x)|^{2} d x,
$$

from which, due to Lemma 1.6, one has the second inequality in (1.7). The proof of the proposition is now complete.

\section{Approximation of a displacement by a rigid body displacement.}

In this section we first show that for some classes of star-shaped domains, one can approximate an $H^{1}-$ displacement by a rigid body one. This result can be applied to curved rods. To do so, we prove in Lemma 2.3 below, that any sufficiently small portion of a curved rod as defined in Definition 1.2, is star-shaped.

In the sequel, the Einstein summation convention on repeated indices $i, j \in\{1,2,3\}$ is adopted.

Let $\Omega$ be an open set of $\mathbb{R}^{3}$ and $u$ a displacement belonging to $H^{1}\left(\Omega ; \mathbb{R}^{3}\right)$. The deformation energy of $u$ is defined by

$$
\mathcal{E}(u, \Omega)=\int_{\Omega} \gamma_{i j}(u) \gamma_{i j}(u) d x
$$

where

$$
\gamma_{i j}(u)=\frac{1}{2}\left(\frac{\partial u_{i}}{\partial x_{j}}+\frac{\partial u_{j}}{\partial x_{i}}\right),
$$

are the components of the the linearized strain tensor $\gamma=\left(\gamma_{i j}\right)_{1 \leq i, j \leq 3}$. We also introduce the bilinear form

$$
\mathcal{D}(u, \Omega)=\int_{\Omega} \frac{\partial u_{i}}{\partial x_{j}} \frac{\partial u_{i}}{\partial x_{j}} d x .
$$

Lemma 2.1. Let $\Omega$ be a bounded open set of diameter $R$, star-shaped with respect to $B\left(O ; R_{1}\right)$. For any displacement $u \in H^{1}\left(\Omega ; \mathbb{R}^{3}\right)$, let us consider the rigid body displacement $r$ defined by

$$
r(x)=A+B \wedge x,
$$


where

$$
A=\frac{3}{4 \pi R_{1}^{3}} \int_{B\left(O ; R_{1}\right)} u(x) d x, \quad B=\frac{15}{8 \pi R_{1}^{5}} \int_{B\left(O ; R_{1}\right)} x \wedge u(x) d x
$$

Then, the following estimates hold:

$$
\left\{\begin{array}{l}
\mathcal{D}(u-r, \Omega) \leq C\left(\frac{R}{R_{1}}\right)^{13} \mathcal{E}(u, \Omega), \\
\|u-r\|_{L^{2}\left(\Omega ; \mathbb{R}^{3}\right)}^{2} \leq C\left(\frac{R}{R_{1}}\right)^{13} R^{2} \mathcal{E}(u, \Omega) .
\end{array}\right.
$$

Remark 2.2. The first inequality in (2.3) is the Korn inequality corresponding to $\Omega$. The coefficient $\left(R / R_{1}\right)^{13}$ is sharp.

Proof of Lemma 2.1. Following [2] or [9], let $v \in H_{0}^{1}\left(\Omega ; \mathbb{R}^{3}\right)$ be the solution of the problem

$$
\begin{cases}\Delta v=\Delta u & \text { in } \Omega, \\ v=0 & \text { on } \partial \Omega .\end{cases}
$$

One knows that $w=u-v$ belongs to $H_{\text {loc }}^{2}\left(\Omega ; \mathbb{R}^{3}\right) \cap H^{1}\left(\Omega ; \mathbb{R}^{3}\right)$. Moreover, the following Korn inequality holds (see [2] or [9]:

$$
\left\{\begin{array}{l}
\int_{\Omega} \rho^{2}(x) \sum_{i, j, l=1}^{3}\left|\frac{\partial^{2} w_{i}}{\partial x_{j} \partial x_{l}}\right|^{2} d x \leq C \mathcal{E}(u, \Omega), \\
\mathcal{D}(v, \Omega) \leq C \mathcal{E}(v, \Omega) \leq C \mathcal{E}(u, \Omega)
\end{array}\right.
$$

where $\rho(x)$ is the distance from $x$ to $\partial \Omega$.

Applying Poincaré-Wirtinger inequality (1.7) to the components of gradient of $\nabla w$ and using inequality (2.4), we get the following estimate:

$$
\int_{\Omega} \sum_{i, j=1}^{3}\left|\frac{\partial u_{i}}{\partial x_{j}}-\mathcal{M}_{\Omega}\left(\frac{\partial u_{i}}{\partial x_{j}}\right)\right|^{2} d x \leq C\left(\frac{R}{R_{1}}\right)^{4} \mathcal{E}(u, \Omega)
$$

Let $a=\mathcal{M}_{\Omega}(u)$ and $\mathcal{B}$ be the skew-symmetric part of the matrix $\left(\mathcal{M}_{\Omega}\left(\frac{\partial u_{i}}{\partial x_{j}}\right)\right)_{1 \leq i, j \leq 3}$. Introduce also the vector $b \in \mathbb{R}^{3}$ such that

$$
r_{1}(x)=a+\mathcal{B} \cdot x=a+b \wedge x .
$$

Using (2.5) and applying again the Poincaré-Wirtinger inequality to $u-r_{1}$, we obtain

$$
\left\{\begin{array}{l}
\mathcal{D}\left(u-r_{1}, \Omega\right) \leq C\left(\frac{R}{R_{1}}\right)^{13} \mathcal{E}(u, \Omega), \\
\left\|u-r_{1}\right\|_{L^{2}\left(\Omega ; \mathbb{R}^{3}\right)}^{2} \leq C\left(\frac{R}{R_{1}}\right)^{8} R^{2} \mathcal{E}(u, \Omega) .
\end{array}\right.
$$

To derive estimate (2.3), it is now sufficient to take in (2.6) the mean value of $u$ and of $(\cdot \wedge u(\cdot))$ in the ball $B\left(O ; R_{1}\right)$.

Let now consider a partition of the rod $\mathcal{P}_{\delta}$ in several small portions of the form $\mathcal{P}_{\delta}\left(t, t^{\prime}\right)$ (for notation, see (1.4) and (1.5) in Definition 1.1). We prove that all these portions are star-shaped with respect to an 
appropriate ball. This will allow us to apply Lemma 2.1 to a curved rod in order to obtain an approximation of its displacements by a discrete family of rigid body displacements.

Lemma 2.3. Let $t, t^{\prime}$ such that $0 \leq t<t^{\prime} \leq L$. The domain $\mathcal{P}_{\delta}\left(t, t^{\prime}\right)$ is star-shaped with respect to the ball $B\left(M\left(\frac{t+t^{\prime}}{2}\right) ; \frac{\delta}{5}\right)$ if

$$
\delta \leq t^{\prime}-t \leq \frac{3 \delta}{2}
$$

Proof. Observe that the diameter of $\mathcal{P}_{\delta, t, t^{\prime}}$ is smaller than $4 \delta$. Since, due to Hypothesis 1.1, the Frenet frame is sufficiently smooth, by Taylor inequality we have

$$
\left\{\begin{array}{l}
\left\|\overrightarrow{M(s) M\left(s^{\prime}\right)}\right\|_{\mathbb{R}^{3}} \leq\left|s^{\prime}-s\right|, \\
\left.\left\|\overrightarrow{M(s) M\left(s^{\prime}\right)}-\left(s^{\prime}-s\right) \vec{T}(s)\right\|_{\mathbb{R}^{3}} \leq \frac{1}{2\|c\|_{L^{\infty}(0, L)}}\left(s^{\prime}-s\right)^{2}, \quad \forall s, s^{\prime} \in\right] 0, L\left[^{2} .\right.
\end{array}\right.
$$

Let $K=K(s, \theta)$ be a point of the lateral boundary of $\mathcal{P}_{\delta}\left(t, t^{\prime}\right)$. The above inequality implies that the distance from $K$ to the point $M\left(\frac{t+t^{\prime}}{2}\right)$, satisfies, since $\delta<\delta_{0}$.

$$
\left\|\overrightarrow{M\left(\frac{t+t^{\prime}}{2}\right) K}\right\|_{\mathbb{R}^{3}} \geq\left(\left|s-\frac{t+t^{\prime}}{2}\right|^{2}+\delta^{2}\right)^{1 / 2}-\frac{1}{2\|c\|_{L^{\infty}(0, L)}}\left|s-\frac{t+t^{\prime}}{2}\right|^{2} \geq \delta .
$$

If $K$ belongs to one of the discs $D(M(t) ; \delta)$ and $D\left(M\left(t^{\prime}\right) ; \delta\right)$, then

$$
\left\|\overrightarrow{M\left(\frac{t+t^{\prime}}{2}\right) K}\right\|_{\mathbb{R}^{3}} \geq\left|\frac{t^{\prime}-t}{2}\right|-\frac{1}{2\|c\|_{L^{\infty}(0, L)}}\left|\frac{t^{\prime}-t}{2}\right|^{2} \geq \frac{2 \delta}{5} .
$$

The ball $B(M(s) ; \delta), t<s<t^{\prime}$, contains the ball $B_{0}=B\left(M\left(\frac{t+t^{\prime}}{2}\right) ; \frac{\delta}{5}\right)$ because the distance between their centers is smaller than $3 \delta / 4$. From the above inequalities, we know that the distance between $M(s)$ and any point on the lateral boundary of $\mathcal{P}_{\delta}\left(t, t^{\prime}\right)$ is greater than or equal to $\delta$. Therefore, the intersection of the ball $B(M(s) ; \delta)$ with the boundary of $\mathcal{P}_{\delta}\left(t, t^{\prime}\right)$ is included in the discs $D(M(t) ; \delta)$ and $D\left(M\left(t^{\prime}\right) ; \delta\right)$. Thus, $B(M(s) ; \delta) \cap \mathcal{P}_{\delta}\left(t, t^{\prime}\right)$ is a convex open set containing $B_{0}$ and so, it is star-shaped with respect to all points of $B_{0}$. But any point of $\mathcal{P}_{\delta}\left(t, t^{\prime}\right)$ belongs to a ball of radius $\delta$ and of center the point $M(s)$ with $t<s<t^{\prime}$. Consequently, the domain $\mathcal{P}_{\delta}\left(t, t^{\prime}\right)$ is star-shaped with respect to the ball $B_{0}$ and this ends the proof of Lemma 2.3.

\section{Elementary displacements of a curved rod.}

Definition 3.1. We call elementary displacement of a rod, any element $\eta$ of $H^{1}\left(\omega_{\delta} ; \mathbb{R}^{3}\right)$ that is written in the form

$$
\eta\left(s, s_{2}, s_{3}\right)=\mathcal{A}(s)+\mathcal{B}(s) \wedge\left(s_{2} \vec{N}(s)+s_{3} \vec{B}(s)\right), \quad \forall\left(s, s_{2}, s_{3}\right) \in \omega_{\delta}
$$

where $\mathcal{A}$ and $\mathcal{B}$ are vector-functions in $H^{1}\left(0, L ; \mathbb{R}^{3}\right)$.

The first component $\mathcal{A}$ of $\eta$ is the displacement of the middle-line. The second component $\mathcal{B}$, gives us an information about the relative displacement of the cross section $D(M(s) ; \delta)$ of the rod, that is to say, it is a rotation whose axis is directed along the vector $\mathcal{B}(s)$.

To any displacement $w$ of the rod we associate an elementary displacement defined as follows: 
Definition 3.2. The elementary displacement $W_{e}$, associated to $w \in H^{1}\left(\mathcal{P}_{\delta} ; \mathbb{R}^{3}\right)$, is given by

$$
W_{e}\left(s, s_{2}, s_{3}\right)=\mathcal{W}(s)+\mathcal{R}_{w}(s) \wedge\left(s_{2} \vec{N}(s)+s_{3} \vec{B}(s)\right), \quad\left(s, s_{2}, s_{3}\right) \in \omega_{\delta},
$$

where

$$
\left\{\begin{array}{l}
\mathcal{W}(s)=\frac{1}{\pi \delta^{2}} \int_{D(O ; \delta)} w\left(s, s_{2}, s_{3}\right) d s_{2} d s_{3} \\
\mathcal{R}_{w}(s) \cdot \vec{T}(s)=\frac{2}{\pi \delta^{4}} \int_{D(O ; \delta)}\left[\left(s_{2} \vec{N}(s)+s_{3} \vec{B}(s)\right) \wedge w\left(s, s_{2}, s_{3}\right)\right] \cdot \vec{T}(s) d s_{2} d s_{3} \\
\mathcal{R}_{w}(s) \cdot \vec{N}(s)=\frac{4}{\pi \delta^{4}} \int_{D(O ; \delta)}\left[\left(s_{2} \vec{N}(s)+s_{3} \vec{B}(s)\right) \wedge w\left(s, s_{2}, s_{3}\right)\right] \cdot \vec{N}(s) d s_{2} d s_{3} \\
\mathcal{R}_{w}(s) \cdot \vec{B}(s)=\frac{4}{\pi \delta^{4}} \int_{D(O ; \delta)}\left[\left(s_{2} \vec{N}(s)+s_{3} \vec{B}(s)\right) \wedge w\left(s, s_{2}, s_{3}\right)\right] \cdot \vec{B}(s) d s_{2} d s_{3} .
\end{array}\right.
$$

The next theorem plays a fundamental role in our study. It provides sharp estimates of the components of the elementary displacement $W_{e}$ in terms of $\delta$ and of the energy of deformation of $w$. It also shows that the displacement $w-W_{e}$ is negligible (in the $L^{2}-$ norm) with respect to $w$.

Theorem 3.3. Let $w \in H^{1}\left(\mathcal{P}_{\delta} ; \mathbb{R}^{3}\right)$ and $W_{e}$ be the elementary displacement defined by (3.1)-(3.2). Then, the following estimates hold:

$$
\left\{\begin{array}{l}
\mathcal{D}\left(w-W_{e}, \omega_{\delta}\right) \leq C \mathcal{E}\left(w, \mathcal{P}_{\delta}\right) \\
\left\|w-W_{e}\right\|_{L^{2}\left(\omega_{\delta} ; \mathbb{R}^{3}\right)}^{2} \leq C \delta^{2} \mathcal{E}\left(w, \mathcal{P}_{\delta}\right), \\
\delta^{2}\left\|\frac{d \mathcal{R}_{w}}{d s}\right\|_{L^{2}\left(0, L ; \mathbb{R}^{3}\right)}^{2}+\left\|\frac{d \mathcal{W}}{d s}-\mathcal{R}_{w} \wedge \vec{T}\right\|_{L^{2}\left(0, L ; \mathbb{R}^{3}\right)}^{2} \leq \frac{C}{\delta^{2}} \mathcal{E}\left(w, \mathcal{P}_{\delta}\right) .
\end{array}\right.
$$

The constants are independent of $\delta$, they depend only on the middle-line of the rod.

Proof We are led in the course of the proof, to take integrals over the domains $\mathcal{P}_{\delta}, \omega_{\delta}$ or on subsets of these domains and estimate them in terms of $w$. To compute these integrals, one has to make the change of variables $\Phi$, defined in (1.3). Observe that the jacobian $\mathcal{J}(\Phi)$ of $\Phi$ is such that

$$
\frac{1}{2}<\mathcal{J}(\Phi)<\frac{3}{2}
$$

This will allow us to neglect it in all the estimates below.

Let $N$ be an integer such that

$$
N \in\left(\frac{2 L}{3 \delta}, \frac{L}{\delta}\right)
$$

and set

$$
s_{p}=p \frac{L}{N}, \quad p \in\{0, \ldots, N\}
$$

In the sequel we will work with portions of the $\operatorname{rod} \mathcal{P}_{\delta}$ of the form $\mathcal{P}_{\delta}\left(s_{p}, s_{p+1}\right)$, that for the sake of simplicity, will be denoted $\mathcal{P}_{\delta, p}$,

$$
\mathcal{P}_{\delta}\left(s_{p}, s_{p+1}\right)=\mathcal{P}_{\delta, p}
$$

From Lemma 2.1 and Lemma 2.3, there exist rigid body displacements $r_{p}$ such that

$$
\left\{\begin{array}{l}
\mathcal{D}\left(w-r_{p}, \mathcal{P}_{\delta, p}\right) \leq C \mathcal{E}\left(w, \mathcal{P}_{\delta, p}\right) \\
\left\|w-r_{p}\right\|_{L^{2}\left(\mathcal{P}_{\delta, p} ; \mathbb{R}^{3}\right)}^{2} \leq C \delta^{2} \mathcal{E}\left(w, \mathcal{P}_{\delta, p}\right),
\end{array}\right.
$$


where the constants are independ of $p$ and $\delta$. Recall that the displacements $r_{p}$ are of the form

$$
r_{p}(x)=A_{p}+B_{p} \wedge x, \quad x \in \mathcal{P}_{\delta, p}, \quad A_{p}, B_{p} \in \mathbb{R}^{3} .
$$

Taking the mean value over the cross-section of the curved rod, and using definition (3.2) of $\mathcal{W}$ and $\mathcal{R}_{w}$, from (3.4) we derive the estimates

$$
\left\{\begin{array}{l}
\left\|\mathcal{W}-A_{p}-B_{p} \wedge \overrightarrow{O M}(\cdot)\right\|_{L^{2}\left(p \varepsilon,(p+1) \varepsilon ; \mathbb{R}^{3}\right)}^{2} \leq C \mathcal{E}\left(w, \mathcal{P}_{\delta, p}\right) \\
\left\|\mathcal{R}_{w}-B_{p}\right\|_{L^{2}\left(p \varepsilon,(p+1) \varepsilon ; \mathbb{R}^{3}\right)}^{2} \leq \frac{C}{\delta^{2}} \mathcal{E}\left(w, \mathcal{P}_{\delta, p}\right)
\end{array}\right.
$$

where the constants are again independent of $p$ and $\delta$. Consequently,

$$
\left\|w-W_{e}\right\|_{L^{2}\left(\omega_{\delta} ; \mathbb{R}^{3}\right)}^{2} \leq C \delta^{2} \mathcal{E}\left(w, \mathcal{P}_{\delta}\right)
$$

where $C$ depends on the middle-line of the rod only.

Note now that the vector-functions $\mathcal{W}$ and $\mathcal{R}_{w}$ belong both to $H^{1}\left(0, L ; \mathbb{R}^{3}\right)$. Then, by using Definition 3.2 as well as definitions (1.1) and (1.2) of the Frenet frame, we compute the derivatives of the displacement $u$ to get

$$
\left\{\begin{array}{l}
\frac{\partial w}{\partial s}=\nabla_{x} w \cdot\left[\left(1-s_{2} c\right) \vec{T}+s_{2} \tau \vec{B}-s_{3} \tau \vec{N}\right] \\
\frac{\partial w}{\partial s_{2}}=\nabla_{x} w \cdot \vec{N} \\
\frac{\partial w}{\partial s_{3}}=\nabla_{x} w \cdot \vec{B}
\end{array}\right.
$$

Taking the restrictions of these derivatives to $\left.\omega_{\delta, p}=\right] p \varepsilon,(p+1) \varepsilon[\times D(O ; \delta)$, from (3.4) we have the estimates

$$
\left\{\begin{array}{l}
\left\|\frac{\partial w}{\partial s}-B_{p} \wedge\left[\left(1-s_{2} c\right) \vec{T}+s_{2} \tau \vec{B}-s_{3} \tau \vec{N}\right]\right\|_{L^{2}\left(\omega_{\delta, p} ; \mathbb{R}^{3}\right)}^{2} \leq C \mathcal{E}\left(w, \mathcal{P}_{\delta, p}\right) \\
\left\|\frac{\partial w}{\partial s_{2}}-B_{p} \wedge \vec{N}\right\|_{L^{2}\left(\omega_{\delta, p} ; \mathbb{R}^{3}\right)}^{2} \leq C \mathcal{E}\left(w, \mathcal{P}_{\delta, p}\right), \\
\left\|\frac{\partial w}{\partial s_{3}}-B_{p} \wedge \vec{B}\right\|_{L^{2}\left(\omega_{\left.\delta, p ; \mathbb{R}^{3}\right)}\right.}^{2} \leq C \mathcal{E}\left(u, \mathcal{P}_{\delta, p}\right),
\end{array}\right.
$$

whence, by taking the mean value on the cross-section of the rod,

$$
\left\|\frac{d \mathcal{W}}{d s}-B_{p} \wedge \vec{T}\right\|_{L^{2}\left(p \varepsilon,(p+1) \varepsilon ; \mathbb{R}^{3}\right)}^{2} \leq \frac{C}{\delta^{2}} \mathcal{E}\left(w, \mathcal{P}_{\delta, p}\right) .
$$

Then, from (3.5) we get

$$
\left\|\frac{d \mathcal{W}}{d s}-\mathcal{R}_{w} \wedge \vec{T}\right\|_{L^{2}\left(0, L ; \mathbb{R}^{3}\right)}^{2} \leq \frac{C}{\delta^{2}} \mathcal{E}\left(w, \mathcal{P}_{\delta}\right)
$$

We now estimate the norm of $\frac{d \mathcal{R}_{w}}{d s}$ in $L^{2}\left(0, L ; \mathbb{R}^{3}\right)$. To begin with, let us introduce the function

$$
V(s)=\frac{4}{\pi \delta^{4}} \int_{D(O ; \delta)}\left(s_{2} \vec{N}(s)+s_{3} \vec{B}(s)\right) \wedge w\left(s, s_{2}, s_{3}\right) d s_{2} d s_{3} .
$$


Using (1.1) and (1.2), we have

$$
\begin{aligned}
\frac{d V}{d s}=\frac{4}{\pi \delta^{4}} \int_{D(O ; \delta)} & {\left[\left(s_{2} \vec{N}+s_{3} \vec{B}\right) \wedge \frac{\partial w}{\partial s}\left(s, s_{2}, s_{3}\right)\right.} \\
+ & \left.\left(-s_{2} c \vec{T}+s_{2} \tau \vec{B}-s_{3} \tau \vec{N}\right) \wedge w\left(s, s_{2}, s_{3}\right)\right] d s_{2} d s_{3}
\end{aligned}
$$

From (3.4) and using estimate (3.7) for $\frac{\partial w}{\partial s}$, it follows that

$$
\left\|\frac{d V}{d s}-c\left(B_{p} \cdot \vec{N}\right) \vec{T}-c\left(B_{p} \cdot \vec{T}\right) \vec{N}\right\|_{L^{2}\left(p \varepsilon,(p+1) \varepsilon ; \mathbb{R}^{3}\right)}^{2} \leq \frac{C}{\delta^{4}} \mathcal{E}\left(w, \mathcal{P}_{\delta, p}\right),
$$

whence, due to (3.5),

$$
\left\|\frac{d V}{d s}-c\left(\mathcal{R}_{w} \cdot \vec{N}\right) \vec{T}-c\left(\mathcal{R}_{w} \cdot \vec{T}\right) \vec{N}\right\|_{L^{2}\left(0, L ; \mathbb{R}^{3}\right)}^{2} \leq \frac{C}{\delta^{4}} \mathcal{E}\left(w, \mathcal{P}_{\delta}\right) .
$$

Using the definitions of $\mathcal{R}_{w}$ and $V$, one can write

$$
\mathcal{R}_{w}=V-\frac{1}{2}(V \cdot \vec{T}) \vec{T}
$$

and so,

$$
\frac{d \mathcal{R}_{w}}{d s}=\frac{d V}{d s}-\frac{1}{2}\left(\frac{d V}{d s} \cdot \vec{T}\right) \vec{T}-\frac{c}{2}(V \cdot \vec{N}) \vec{T}-\frac{c}{2}(V \cdot \vec{T}) \vec{N} .
$$

Since $(V \cdot \vec{N})=\left(\mathcal{R}_{w} \cdot \vec{N}\right)$ and $(V \cdot \vec{T})=2\left(\mathcal{R}_{w} \cdot \vec{T}\right)$, recalling (3.9), we deduce that

$$
\left\|\frac{d \mathcal{R}_{w}}{d s}\right\|_{L^{2}\left(0, L ; \mathbb{R}^{3}\right)}^{2} \leq \frac{C}{\delta^{4}} \mathcal{E}\left(w, \mathcal{P}_{\delta}\right) .
$$

Now, observe that

$$
\begin{aligned}
\frac{\partial}{\partial s}\left(w-W_{e}\right) & =\frac{\partial w}{\partial s}-\frac{\partial \mathcal{W}}{\partial s}-\frac{\partial \mathcal{R}_{w}}{\partial s} \wedge\left(s_{2} \vec{N}+s_{3} \vec{B}\right)-\mathcal{R}_{w} \wedge\left(-s_{2} c \vec{T}+s_{2} \tau \vec{B}-s_{3} \tau \vec{N}\right), \\
\frac{\partial}{\partial s_{2}}\left(w-W_{e}\right) & =\frac{\partial w}{\partial s_{2}}-\mathcal{R}_{w} \wedge \vec{N} \\
\frac{\partial}{\partial s_{3}}\left(w-W_{e}\right) & =\frac{\partial w}{\partial s_{3}}-\mathcal{R}_{w} \wedge \vec{B} .
\end{aligned}
$$

From these expressions, taking into account (3.5), (3.7), (3.8) and (3.10), we finally obtain the first estimate in (3.4) and this ends the proof of the theorem.

From now on, we will assume that the curved rod $\mathcal{P}_{\delta}$ is clamped at the end

$$
\Gamma_{0 \delta}=\Phi(\{0\} \times D(M(0) ; \delta)) .
$$

Then, the space of admissible displacements of the rod is

$$
H_{\Gamma_{0}}^{1}\left(\mathcal{P}_{\delta} ; \mathbb{R}^{3}\right)=\left\{v \in H^{1}\left(\mathcal{P}_{\delta} ; \mathbb{R}^{3}\right) \mid v=0 \text { on } \Gamma_{0 \delta}\right\},
$$

equipped with the semi-norm

$$
|v|_{\mathcal{E}}=\sqrt{\mathcal{E}\left(v, \mathcal{P}_{\delta}\right)}
$$

According to estimates from Theorem 3.3, and since the elementary displacement $V_{e}$ associated to any $v \in H_{\Gamma_{0}}^{1}\left(\mathcal{P}_{\delta} ; \mathbb{R}^{3}\right)$ is equal to zero on the clamped part of the rod, the semi-norm (3.11) is actually a norm. Consequently, the space $H_{\Gamma_{0}}^{1}\left(\mathcal{P}_{\delta} ; \mathbb{R}^{3}\right)$ equipped with the norm $|\cdot|_{\mathcal{E}}$ is a Hilbert one. 


\section{Inextensional and extensional displacements, angle of torsion.}

Let us introduce now two sets of displacements of a $\operatorname{rod} \mathcal{P}_{\delta}$.

Definition 4.1. The set of inextensional displacements of the middle-line of the rod is defined as follows:

$$
D_{\text {In }}=\left\{U \in H^{1}\left(0, L ; \mathbb{R}^{3}\right) \mid \frac{d U}{d s} \cdot \vec{T}=0, \quad U(0)=0\right\}
$$

The set of the extensional displacements of the middle-line of the rod is defined by

$$
D_{E x}=\left\{U \in H^{1}\left(0, L ; \mathbb{R}^{3}\right) \mid \frac{d U}{d s} \cdot \vec{N}=\frac{d U}{d s} \cdot \vec{B}=0, \quad U(0)=0\right\}
$$

Observe that by definition, for any $U \in D_{E x}$, one has

$$
\left\|\frac{d U}{d s}\right\|_{L^{2}\left(0, L ; \mathbb{R}^{3}\right)}=\left\|\frac{d U}{d s} \cdot \vec{T}\right\|_{L^{2}(0, L)} .
$$

Let $w \in H_{\Gamma_{0}}^{1}\left(\mathcal{P}_{\delta} ; \mathbb{R}^{3}\right)$, and $W_{e}$ be the associated elementary displacement as defined in (3.1) and (3.2). Obviously, $W_{e} \in H_{\Gamma_{0}}^{1}\left(\mathcal{P}_{\delta} ; \mathbb{R}^{3}\right)$ too. Its first component $\mathcal{W}$, which is the displacement of the middle-line, can be written as the sum of an inextensional displacement and an extensional one, i.e.

$$
\mathcal{W}=W_{I}+W_{E}, \quad W_{I} \in D_{I n}, \quad W_{E} \in D_{E x}
$$

From (3.3), definitions (4.1) and (4.2), the following a priori estimates are obvious:

$$
\left\{\begin{array}{l}
\left\|W_{I}\right\|_{H^{1}\left(0, L ; \mathbb{R}^{3}\right)}+\left\|\frac{d W_{I}}{d s}\right\|_{L^{2}\left(0, L ; \mathbb{R}^{3}\right)} \leq \frac{C}{\delta^{2}}|w|_{\mathcal{E}} \\
\left\|W_{E}\right\|_{H^{1}\left(0, L ; \mathbb{R}^{3}\right)}+\left\|\frac{d W_{E}}{d s}\right\|_{L^{2}\left(0, L ; \mathbb{R}^{3}\right)} \leq \frac{C}{\delta}|w|_{\mathcal{E}}
\end{array}\right.
$$

The second component $\mathcal{R}_{w}$ of $W_{e}$, not only allows us to correct the traction resulting from a shearing effect which depends only on $\mathcal{R}_{w} \wedge \vec{T}$, but it also gives information about the torsion of the rod. This displacement is measured by the angle of torsion of the rod which is given by the inner product of the vector $\mathcal{R}_{w}$ and of the unit tangent vector $\vec{T}$ to the middle-line,

$$
\Theta_{w}=\mathcal{R}_{w} \cdot \vec{T}
$$

If the displacement is small, $\Theta_{w}$ is almost equal to the rotation of the cross section $D(M(s) ; \delta)$ around its axis.

To complete the decomposition of the displacements of the rod, we only need to introduce what we will call "residual" displacements. These displacements will play an essential role in the study of the strain and stress tensors of the rod.

To begin with, observe that any $w$ can be written in the form

$$
\begin{aligned}
w\left(s, s_{2}, s_{3}\right)=\mathcal{W}(s) & +\mathcal{R}_{w}(s) \wedge\left(s_{2} \vec{N}(s)+s_{3} \vec{B}(s)\right) \\
& +\left(w-W_{e}\right)\left(s, s_{2}, s_{3}\right), \quad \forall\left(s, s_{2}, s_{3}\right) \in \omega_{\delta} .
\end{aligned}
$$

On the other hand, using decomposition (4.3) and since

$$
\mathcal{R}_{w}=\Theta_{w} \vec{T}-\frac{d W_{I}}{d s} \wedge \vec{T}+\left(\frac{d W_{I}}{d s}-\mathcal{R}_{w} \wedge \vec{T}\right) \wedge \vec{T}
$$


one can also rewrite $(4.6)$ as

$$
\begin{aligned}
w\left(\cdot, s_{2}, s_{3}\right)= & W_{I}+W_{E}-\left[s_{2}\left(\frac{d W_{I}}{d s} \cdot \vec{N}\right)+s_{3}\left(\frac{d W_{I}}{d s} \cdot \vec{B}\right)\right] \vec{T}+\left(-s_{3} \vec{N}+s_{2} \vec{B}\right) \Theta_{w} \\
& +s_{2}\left(\frac{d W_{I}}{d s}-\mathcal{R}_{w} \wedge \vec{T}\right) \cdot \vec{N}+s_{3}\left(\frac{d W_{I}}{d s}-\mathcal{R}_{w} \wedge \vec{T}\right) \cdot \vec{B}+\left(w-W_{e}\right)\left(\cdot, s_{2}, s_{3}\right) .
\end{aligned}
$$

Let now introduce the following space:

$$
H_{\mathbb{R}^{3}}\left(\left\{\partial_{S_{2}}, \partial_{S_{3}}\right\} ; \omega\right)=\left\{v \in L^{2}\left(\omega ; \mathbb{R}^{3}\right) \mid \frac{\partial v}{\partial S_{2}}, \frac{\partial v}{\partial S_{3}} \in L^{2}\left(\omega ; \mathbb{R}^{3}\right)\right\}
$$

Definition 4.2. Let $w \in H_{\Gamma_{0}}^{1}\left(\mathcal{P}_{\delta} ; \mathbb{R}^{3}\right)$. We call residual displacement associated to $w$, the vector-function $\widehat{w} \in H_{\mathbb{R}^{3}}\left(\left\{\partial_{S_{2}}, \partial_{S_{3}}\right\} ; \omega\right)$ :

$$
\begin{aligned}
\widehat{w}\left(s, S_{2}, S_{3}\right)=\delta S_{2}\left(\frac{d W_{I}}{d s}\right. & \left.-\mathcal{R}_{w} \wedge \vec{T}\right) \cdot \vec{N}+\delta S_{3}\left(\frac{d W_{I}}{d s}-\mathcal{R}_{w} \wedge \vec{T}\right) \cdot \vec{B} \\
& +\left(w-W_{e}\right)\left(s, \delta S_{2}, \delta S_{3}\right), \quad \forall\left(s, S_{2}, S_{3}\right) \in \omega .
\end{aligned}
$$

Definition 4.3. By analogy with the case of a straight rod, we call Bernoulli-Navier displacement of a curved rod, any displacement of the form

$$
\begin{aligned}
v\left(s, s_{2}, s_{3}\right)=V_{I}(s)+ & V_{E}(s)-\left(s_{2} \frac{d V_{I}}{d s}(s) \cdot \vec{N}(s)+s_{3} \frac{d V_{I}}{d s}(s) \cdot \vec{B}(s)\right) \vec{T}(s) \\
& +\left(-s_{3} \vec{N}(s)+s_{2} \vec{B}(s)\right) \cdot \Theta_{v}(s), \quad \forall\left(s, s_{2}, s_{3}\right) \in \omega_{\delta}
\end{aligned}
$$

where $V_{I} \in D_{I n}$ and $V_{E} \in D_{E x}$.

Definitions 4.2 and 4.3 (see also Notation 1.3 ) imply that any displacement $w$ in $H_{\Gamma_{0}}^{1}\left(\mathcal{P}_{\delta} ; \mathbb{R}^{3}\right)$, can be written as the sum of two displacements, a Bernoulli-Navier one and a remainder term

$$
\begin{aligned}
& w\left(\cdot, s_{2}, s_{3}\right)=W_{I}+ W_{E}-\left(s_{2} \frac{d W_{I}}{d s} \cdot \vec{N}+s_{3} \frac{d W_{I}}{d s} \cdot \vec{B}\right) \vec{T} \\
&+\left(-s_{3} \vec{N}+s_{2} \vec{B}\right) \Theta_{w}+\widehat{w}\left(\cdot, \frac{s_{2}}{\delta}, \frac{s_{3}}{\delta}\right), \quad \forall\left(s_{2}, s_{3}\right) \in D(O ; \delta) .
\end{aligned}
$$

From (3.3) it follows that

$$
\|\widehat{w}\|_{L^{2}\left(\omega ; \mathbb{R}^{3}\right)}+\left\|\frac{\partial \widehat{w}}{\partial S_{2}}\right\|_{L^{2}\left(\omega ; \mathbb{R}^{3}\right)}+\left\|\frac{\partial \widehat{w}}{\partial S_{3}}\right\|_{L^{2}\left(\omega ; \mathbb{R}^{3}\right)} \leq C|w|_{\mathcal{E}}
$$

REMARK 4.4. Let us mention that decomposition (4.8) is somewhat "false" in the sense that nor the Bernoulli-Navier displacement neither the residual displacement $\widehat{w}$ are admissible displacements (see (3.5)) of the curved rod.

On account of the definitions of the different elements in (4.7), it is easily seen that $\widehat{w}$ satisfies the following equalities (see Notation 1.3):

$$
\left\{\begin{array}{l}
\int_{D(O ; 1)} \widehat{w}\left(\cdot, S_{2}, S_{3}\right) d S_{2} d S_{3}=0 \\
\int_{D(O ; 1)} \widehat{w}\left(\cdot, S_{2}, S_{3}\right) \cdot\left[S_{3} \cdot \vec{N}(\cdot)-S_{2} \vec{B}(\cdot)\right] d S_{2} d S_{3}=0
\end{array}\right.
$$


The set $\mathcal{V}_{m}$ of the residual displacements,

$$
\mathcal{V}_{m}=\left\{\psi \in H_{\mathbb{R}^{3}}\left(\left\{\partial_{S_{2}}, \partial_{S_{3}}\right\} ; \omega\right) \mid \psi \text { satisfies } \quad(4.10)\right\}
$$

is a a Hilbert space for the norm

$$
\|\psi\|_{m}=\left(\left\|\frac{\partial \psi}{\partial S_{2}}\right\|_{L^{2}\left(\omega ; \mathbb{R}^{3}\right)}^{2}+\left\|\frac{\partial \psi}{\partial S_{3}}\right\|_{L^{2}\left(\omega ; \mathbb{R}^{3}\right)}^{2}\right)^{1 / 2} .
$$

\section{Limit of displacements and of the strain tensor.}

\subsection{Limit of displacements}

Suppose we are given a sequence $\left(w^{\delta}\right) \subset H_{\Gamma_{0}}^{1}\left(\mathcal{P}_{\delta} ; \mathbb{R}^{3}\right)$. Applying the results from Section 4 , we can decompose $w^{\delta}$ in a sum of displacements of the form (4.8) as follows:

$$
\begin{aligned}
w^{\delta}\left(s, s_{2}, s_{3}\right)= & W_{I}^{\delta}(s)+W_{E}^{\delta}(s)-\left(s_{2} \frac{d W_{I}^{\delta}}{d s}(s) \cdot \vec{N}(s)+s_{3} \frac{d W_{I}^{\delta}}{d s}(s) \cdot \vec{B}(s)\right) \vec{T}(s) \\
& +\left(-s_{3} \vec{N}(s)+s_{2} \vec{B}(s)\right) \Theta_{w}^{\delta}(s)+\widehat{w}^{\delta}\left(s, \frac{s_{2}}{\delta}, \frac{s_{3}}{\delta}\right), \quad \forall\left(s, s_{2}, s_{3}\right) \in \omega_{\delta}
\end{aligned}
$$

where $W_{I}^{\delta} \in D_{I n}, W_{E}^{\delta} \in D_{E x}$ and

$$
\begin{aligned}
\widehat{w}^{\delta}\left(\cdot, \frac{s_{2}}{\delta}, \frac{s_{3}}{\delta}\right)=s_{2}\left(\frac{d W_{I}^{\delta}}{d s}\right. & \left.-\mathcal{R}_{w}^{\delta} \wedge \vec{T}\right) \cdot \vec{N}+s_{3}\left(\frac{d W_{I}^{\delta}}{d s}-\mathcal{R}_{w}^{\delta} \wedge \vec{T}\right) \cdot \vec{B} \\
& +\left(w^{\delta}-W_{e}^{\delta}\right)\left(\cdot, s_{2}, s_{3}\right), \quad \forall\left(s_{2}, s_{3}\right) \in D(O ; \delta) .
\end{aligned}
$$

We are interested to describe the behaviour of the sequence $\left(w^{\delta}\right)$ when $\delta \rightarrow 0$. The next result answers this question:

Proposition 5.1. Suppose that for any $\delta>0$, one has the estimate

$$
\left|w^{\delta}\right|_{\mathcal{E}} \leq C \delta
$$

where the constant $C$ is independent from $\delta$. Then (up to a subsequence), the following convergences hold:

$$
\begin{cases}\text { (i) } & W_{E}^{\delta} \rightarrow W_{E} \quad \text { weakly in } H^{1}\left(0, L ; \mathbb{R}^{3}\right) \\ \text { (ii) } & \delta W_{I}^{\delta} \rightarrow W_{I} \quad \text { weakly in } H^{1}\left(0, L ; \mathbb{R}^{3}\right) \\ \text { (iii) } & \delta \mathcal{R}_{w}^{\delta} \rightarrow \mathcal{R}_{w}^{\star} \quad \text { weakly in } H^{1}\left(0, L ; \mathbb{R}^{3}\right) \\ \text { (iv) } & \frac{1}{\delta} \widehat{w}^{\delta} \rightarrow \widehat{w} \quad \text { weakly in } \mathcal{V}_{m}, \\ \text { (v) } & \delta \mathcal{T}_{\delta}\left(w^{\delta}\right) \rightarrow W_{I} \quad \text { weakly in } H^{1}\left(\omega ; \mathbb{R}^{3}\right) \\ \text { (vi }) & \mathcal{T}_{\delta}\left(w^{\delta}\right)-W_{I}^{\delta} \rightarrow W^{\star} \quad \text { weakly in } H^{1}\left(\omega ; \mathbb{R}^{3}\right)\end{cases}
$$

where $\mathcal{V}_{m}$ is defined by (4.11) and

$$
W^{\star}=W_{E}-\left[S_{2}\left(\frac{d W_{I}}{d s} \cdot \vec{N}\right)+S_{3}\left(\frac{d W_{I}}{d s} \cdot \vec{B}\right)\right] \vec{T}+\left(-S_{3} \vec{N}+S_{2} \vec{B}\right) \Theta_{w}^{\star},
$$

with

$$
\Theta_{w}^{\star}=\mathcal{R}_{w}^{\star} \cdot \vec{T} .
$$


Proof. Thanks to hypothesis (5.3), from Theorem 3.3, estimates (4.4) and (4.9), we immediately have

$$
\left\|W_{E}^{\delta}\right\|_{H^{1}\left(0, L ; \mathbb{R}^{3}\right)}+\delta\left\|W_{I}^{\delta}\right\|_{H^{1}\left(0, L ; \mathbb{R}^{3}\right)}+\delta\left\|\mathcal{R}_{w}^{\delta}\right\|_{H^{1}\left(0, L ; \mathbb{R}^{3}\right)}+\frac{1}{\delta}\left\|\widehat{w}^{\delta}\right\|_{m} \leq C,
$$

from which convergences (5.4)(i)-(iv) are straightforward. Moreover, since by definition (4.5), $\Theta_{w}^{\delta}=\mathcal{R}_{w}^{\delta} \cdot \vec{T}$, one also has

$$
\delta \Theta_{w}^{\delta} \rightarrow \Theta_{w}^{\star} \quad \text { weakly in } H^{1}(0, L)
$$

with $\Theta_{w}^{\star}$ verifying (5.5).

Now, let us turn to formula (5.2). Recalling that

$$
W_{e}^{\delta}\left(s, s_{2}, s_{3}\right)=W_{I}^{\delta}(s)+W_{E}^{\delta}(s)+\mathcal{R}_{w}^{\delta}(s) \wedge\left(s_{2} \vec{N}(s)+s_{3} \vec{B}(s)\right), \quad\left(s, s_{2}, s_{3}\right) \in \omega_{\delta},
$$

by using estimate (5.6), from (5.2) we obtain that

$$
\left\|\mathcal{T}_{\delta}\left(w^{\delta}\right)-W_{I}^{\delta}\right\|_{H^{1}\left(\omega ; \mathbb{R}^{3}\right)} \leq C
$$

which, together with (5.4)(ii) implies convergence (5.4)(v). Convergence (5.4)(vi) follows simply by passing to the limit in (5.1) and using the former convergences.

Remark 5.2. From (3.8), one gets

$$
\left\|\frac{d W_{I}^{\delta}}{d s}+\frac{d W_{E}^{\delta}}{d s}-\mathcal{R}_{w}^{\delta} \wedge \vec{T}\right\|_{L^{2}\left(0, L ; \mathbb{R}^{3}\right)}^{2} \leq C,
$$

whence, due to (5.4)(i)-(iii)

$$
\frac{d W_{I}}{d s}=\mathcal{R}_{w}^{\star} \wedge \vec{T} .
$$

Consequently, $W_{I} \in \mathcal{D}_{I n}$ where (see Definition 4.1 for comparison)

$$
\mathcal{D}_{\text {In }}=\left\{U \in H^{2}\left(0, L ; \mathbb{R}^{3}\right) \mid \frac{d U}{d s} \cdot \vec{T}=0, \quad U(0)=0, \quad \frac{d U}{d s}(0)=0\right\} .
$$

Also $W_{E} \in D_{E x}$.

Remark 5.3. Proposition 5.1 shows that the limit behaviour of $w_{\delta}$ when $\delta \rightarrow 0$, is described by the triple $\left(W_{I}, W^{\star}, \widehat{w}\right)$, where $W_{I}$ describes the flexion of the rod, $W^{\star}$ its shearing and torsion and $\widehat{w}$ the deformation of the cross-section. In view of (5.4), to this triple correspond three scales in $\delta$, namely $\delta^{-1}, \delta^{0}$ and $\delta^{1}$. This means that for $\delta$ small enough,

$$
w^{\delta}\left(s, s_{2}, s_{3}\right) \sim \frac{1}{\delta} W_{I}(s)+W^{\star}\left(s, \frac{s_{2}}{\delta}, \frac{s_{3}}{\delta}\right)+\delta \widehat{w}\left(s, \frac{s_{2}}{\delta}, \frac{s_{3}}{\delta}\right), \quad \forall\left(s, s_{2}, s_{3}\right) \in \omega_{\delta} .
$$

\subsection{Limit of the strain tensor.}


We are now interested to describe the asymptotic behaviour of the strain tensor $\gamma\left(w^{\delta}\right)$. To do so, we have to write this tensor in the Frenet basis (1.1)-(1.2). Let us denote the transformed tensor by $e=\left(e_{i j}\right)_{1 \leq i, j \leq 3}$. Recalling formulae (3.6), we have

$$
\begin{aligned}
& e_{11}\left(w^{\delta}\right)=\frac{\partial w^{\delta}}{\partial s} \cdot \vec{T}+\frac{1}{1-s_{2} c}\left[s_{2} c \frac{\partial w^{\delta}}{\partial s}+s_{3} \tau \frac{\partial w^{\delta}}{\partial s_{2}}-s_{2} \tau \frac{\partial w^{\delta}}{\partial s_{3}}\right] \cdot \vec{T} \\
& e_{12}\left(w^{\delta}\right)=\frac{1}{2}\left[\frac{\partial w^{\delta}}{\partial s_{2}} \cdot \vec{T}+\frac{\partial w^{\delta}}{\partial s} \cdot \vec{N}\right]+\frac{1}{2\left(1-s_{2} c\right)}\left[s_{2} c \frac{\partial w^{\delta}}{\partial s}+s_{3} \tau \frac{\partial w^{\delta}}{\partial s_{2}}-s_{2} \tau \frac{\partial w^{\delta}}{\partial s_{3}}\right] \cdot \vec{N}, \\
& e_{13}\left(w^{\delta}\right)=\frac{1}{2}\left[\frac{\partial w^{\delta}}{\partial s_{3}} \cdot \vec{T}+\frac{\partial w^{\delta}}{\partial s} \cdot \vec{B}\right]+\frac{1}{2\left(1-s_{2} c\right)}\left[s_{2} c \frac{\partial w^{\delta}}{\partial s}+s_{3} \tau \frac{\partial w^{\delta}}{\partial s_{2}}-s_{2} \tau \frac{\partial w^{\delta}}{\partial s_{3}}\right] \cdot \vec{B}, \\
& e_{22}\left(w^{\delta}\right)=\frac{\partial w^{\delta}}{\partial s_{2}} \cdot \vec{N} \\
& e_{33}\left(w^{\delta}\right)=\frac{\partial w^{\delta}}{\partial s_{3}} \cdot \vec{B}, \\
& e_{23}\left(w^{\delta}\right)=\frac{1}{2}\left[\frac{\partial w^{\delta}}{\partial s_{3}} \cdot \vec{N}+\frac{\partial w^{\delta}}{\partial s_{2}} \cdot \vec{B}\right] .
\end{aligned}
$$

Proposition 5.2. Let $\left(w^{\delta}\right) \subset H_{\Gamma_{0}}^{1}\left(\mathcal{P}_{\delta} ; \mathbb{R}^{3}\right)$ be a sequence satisfying (5.3). Then one has the following convergences, all weak in $L^{2}(\omega)$,

$$
\left\{\begin{array}{l}
\mathcal{T}_{\delta}\left(e_{11}\left(w^{\delta}\right)\right) \rightarrow e_{11}=\frac{d W_{E}}{d s} \cdot \vec{T}-\left[S_{2}\left(\frac{d^{2} W_{I}}{d s^{2}} \cdot \vec{N}\right)+S_{3}\left(\frac{d^{2} W_{I}}{d s^{2}} \cdot \vec{B}\right)\right]+c S_{3} \Theta_{w}^{\star} \\
\mathcal{T}_{\delta}\left(e_{12}\left(w^{\delta}\right)\right) \rightarrow e_{12}=\frac{1}{2}\left[-c S_{3}\left(\frac{d W_{I}}{d s} \cdot \vec{B}\right)-S_{3} \frac{d \Theta_{w}^{\star}}{d s}-\tau S_{2} \Theta_{w}^{\star}+\frac{\partial \widehat{w}}{\partial S_{2}} \cdot \vec{T}\right] \\
\mathcal{T}_{\delta}\left(e_{13}\left(w^{\delta}\right)\right) \rightarrow e_{13}=\frac{1}{2}\left[c S_{2}\left(\frac{d W_{I}}{d s} \cdot \vec{B}\right)+S_{2} \frac{d \Theta_{w}^{\star}}{d s}+\tau S_{3} \Theta_{w}^{\star}+\frac{\partial \widehat{w}}{\partial S_{3}} \cdot \vec{T}\right] \\
\mathcal{T}_{\delta}\left(e_{22}\left(w^{\delta}\right)\right) \rightarrow e_{22}=\frac{\partial \widehat{w}}{\partial S_{2}} \cdot \vec{N} \\
\mathcal{T}_{\delta}\left(e_{33}\left(w^{\delta}\right)\right) \rightarrow e_{33}=\frac{\partial \widehat{w}}{\partial S_{3}} \cdot \vec{B} \\
\mathcal{T}_{\delta}\left(e_{23}\left(w^{\delta}\right)\right) \rightarrow e_{23}=\frac{1}{2}\left[\frac{\partial \widehat{w}}{\partial S_{2}} \cdot \vec{B}+\frac{\partial \widehat{w}}{\partial S_{3}} \cdot \vec{N}\right]
\end{array}\right.
$$

where $W_{E}, W_{I}, \Theta_{w}^{\star}$ and $\widehat{w}$ are defined in Proposition 5.1.

Proof. Estimates (5.3) and (5.6) imply that the sequence $\mathcal{T}_{\delta}\left(e_{i j}\left(w^{\delta}\right)\right)$ for any $i, j \in\{1,2,3\}$, is bounded in $L^{2}(\omega)$ which will allow us to pass to convergent subsequences. Let us show for instance the result concerning $e_{11}\left(w^{\delta}\right)$. From (1.2) and (3.6) we have

$$
\begin{aligned}
e_{11}\left(w^{\delta}\right)=\frac{1}{1-c s_{2}} & {\left[\frac{d W_{E}^{\delta}}{d s} \cdot \vec{T}+c s_{2}\left(\frac{d W_{E}^{\delta}}{d s} \cdot \vec{T}\right)-s_{2}\left(\frac{d^{2} W_{I}^{\delta}}{d s^{2}} \cdot \vec{N}\right)\right.} \\
& \left.-s_{3}\left(\frac{d^{2} W_{I}^{\delta}}{d s^{2}} \cdot \vec{B}\right)+c s_{3} \Theta_{w}^{\delta}\right]+\frac{1}{1-c s_{2}}\left[\delta \frac{\partial \widehat{w}^{\delta}}{\partial s}\left(s, \frac{s_{2}}{\delta}, \frac{s_{3}}{\delta}\right) \cdot \vec{T}\right. \\
& \left.+\tau s_{3}\left(\frac{\partial \widehat{u}^{\delta}}{\partial S_{2}}\left(s, \frac{s_{2}}{\delta}, \frac{s_{3}}{\delta}\right) \cdot \vec{T}\right)-\tau s_{2}\left(\frac{\partial \widehat{u}^{\delta}}{\partial S_{3}}\left(s, \frac{s_{2}}{\delta}, \frac{s_{3}}{\delta}\right) \cdot \vec{T}\right)\right] .
\end{aligned}
$$

Due to estimates (5.6), we can pass to the limit and get the first convergence in (5.8). The others are obtained analogously.

The results above concern the limit displacements and the limit of the deformation tensor for any sequence $\left(w^{\delta}\right)$ satisfying (5.3). Our aim is to study the linearized elasticity problem so, from now on, we will be concerned by the asymptotic behaviour of its solution. 


\section{Elasticity problem.}

We suppose we are given a rod $\mathcal{P}_{\delta}$ made from an isotropic and homogeneous material. The displacement $u^{\delta}=\left(u_{1}^{\delta}, u_{2}^{\delta}, u_{3}^{\delta}\right)$ of $\mathcal{P}_{\delta}$, is the solution of the linearized system of elasticity

$$
\left\{\begin{array}{cl}
-\frac{\partial}{\partial x_{j}}\left(a_{i j k h} \frac{\partial u_{k}^{\delta}}{\partial x_{h}}\right)=F_{i}^{\delta} & \text { in } \mathcal{P}_{\delta}, \\
u^{\delta}=0 & \text { on } \Gamma_{0 \delta}, \\
a_{i j k h} \frac{\partial u_{k}^{\delta}}{\partial x_{h}} n_{j}=G_{i}^{\delta} & \text { on } \Gamma_{\delta}=\partial \mathcal{P}_{\delta} \backslash \Gamma_{0 \delta},
\end{array}\right.
$$

where $i=1,2,3$, and the elasticity coefficients $a_{i j k h}$ are defined by

$$
a_{i j k l}=\lambda \delta_{i j} \delta_{k h}+\mu\left(\delta_{i k} \delta_{j h}+\delta_{i h} \delta_{j k}\right)
$$

The constants $\lambda$ and $\mu$ are the material Lamé coefficients, $F^{\delta}$ is the density of volumic forces and $G^{\delta}$ is the density of surface forces. Obviously, the coefficients $a_{i j k h}$ satisfy the hypothesis of coerciveness, i.e., there exists a constant $C_{0}>0$ such that

$$
a_{i j k h} \beta_{i j} \beta_{k h} \geq C_{0} \beta_{i j} \beta_{i j}, \quad \text { for any matrix } \beta=\left(\beta_{i j}\right)_{i, j} \text { with } \beta_{i j}=\beta_{j i} \text {. }
$$

The variational formulation of problem (6.1) is, to find $u^{\delta} \in H_{\Gamma_{0}}^{1}\left(\mathcal{P}_{\delta} ; \mathbb{R}^{3}\right)$ such that

$$
\int_{\mathcal{P}_{\delta}} a_{i j k h} \gamma_{i j}\left(u^{\delta}\right) \gamma_{k h}(v) d x=\int_{\mathcal{P}_{\delta}} F^{\delta} \cdot v d x+\int_{\Gamma_{\delta}} G^{\delta} \cdot v d_{s} x . \quad \forall v \in H_{\Gamma_{0}}^{1}\left(\mathcal{P}_{\delta} ; \mathbb{R}^{3}\right) .
$$

Denote by $\sigma^{\delta}=\left(\sigma_{i j}^{\delta}\right)_{1 \leq i, j \leq 3}$ the stress tensor, defined by

$$
\sigma_{i j}^{\delta}=a_{i j k h} \gamma_{k h}\left(u^{\delta}\right)
$$

System (6.1) can be rewritten under the equivalent form

$$
\begin{cases}\frac{\partial}{\partial x_{j}} \sigma_{i j}^{\delta}=F_{i}^{\delta} & \text { in } \mathcal{P}_{\delta}, \\ u^{\delta}=0 & \text { on } \Gamma_{0 \delta}, \\ \sigma_{i j} n_{j}=G_{i}^{\delta} & \text { on } \Gamma_{\delta}=\partial \mathcal{P}_{\delta} \backslash \Gamma_{0 \delta},\end{cases}
$$

for $i=1,2,3$. Hence, (6.3) can also be rewritten in the form, that will be used in the sequel,

$$
\int_{\mathcal{P}_{\delta}} \sigma_{k h}^{\delta} \gamma_{k h}(v) d x=\int_{\mathcal{P}_{\delta}} F^{\delta} \cdot v d x+\int_{\Gamma_{\delta}} G^{\delta} \cdot v d_{s} x . \quad \forall v \in H_{\Gamma_{0}}^{1}\left(\mathcal{P}_{\delta} ; \mathbb{R}^{3}\right) .
$$

The rod $\mathcal{P}_{\delta}$ is subjected to two different type of forces, volume and surface forces. We will distinguish also the forces which concern all the displacements of the middle-line (mainly inextensional displacements) and those which concern only the extensional displacements. This distinction is visible in the order of size corresponding to these displacements. To do so, recalling (1.6), we suppose that $F^{\delta}$ and $G^{\delta}$ satisfy the following assumption:

$$
\left\{\begin{array}{l}
F^{\delta}\left(s, s_{2}, s_{3}\right)=\delta f\left(s, \frac{s_{2}}{\delta}, \frac{s_{3}}{\delta}\right)+f^{*}\left(s, \frac{s_{2}}{\delta}, \frac{s_{3}}{\delta}\right), \quad \text { with } f, f^{*} \in L^{2}\left(\omega ; \mathbb{R}^{3}\right), \\
G^{\delta}(s, \theta)=\delta^{2} g(s, \theta)+\delta g^{*}(s, \theta), \quad \text { with } g, g^{*} \in L^{2}\left(\partial \omega ; \mathbb{R}^{3}\right) .
\end{array}\right.
$$


Then, classical results, the Lax-Milgram theorem for instance, give the existence and uniqueness of the solution $u^{\delta} \in H_{\Gamma_{0}}^{1}\left(\mathcal{P}_{\delta} ; \mathbb{R}^{3}\right)$ of problem (6.1) (and so, of (6.3) or (6.5)).

Let now introduce the notation

$$
\left\{\begin{aligned}
F_{I}= & \frac{1}{\pi} \int_{D(O ; 1)} f\left(\cdot, S_{2}, S_{3}\right) d S_{2} d S_{3}+\frac{1}{\pi} \int_{0}^{2 \pi} g(\cdot, \theta) d \theta \\
F_{E}= & \frac{1}{\pi} \int_{D(O ; 1)} f^{*}\left(\cdot, S_{2}, S_{3}\right) d S_{2} d S_{3}+\frac{1}{\pi} \int_{0}^{2 \pi} g^{*}(\cdot, \theta) d \theta \\
F_{T}= & \frac{1}{\pi} \int_{D(O ; 1)}\left(f_{B}^{*}\left(\cdot, S_{2}, S_{3}\right) S_{2}-f_{N}^{*}\left(\cdot, S_{2}, S_{3}\right) S_{3}\right) d S_{2} d S_{3} \\
& +\frac{1}{\pi} \int_{0}^{2 \pi}\left(g_{B}^{*}(\cdot, \theta) \cos (\theta)-g_{N}^{*}(\cdot, \theta) \sin \theta\right) d \theta \\
G_{N}= & \frac{1}{\pi} \int_{D(O ; 1)} f_{T}^{*}\left(\cdot, S_{2}, S_{3}\right) S_{2} d S_{2} d S_{3}+\frac{1}{\pi} \int_{0}^{2 \pi} g_{T}^{*}(\cdot, \theta) \cos \theta d \theta \\
G_{B}= & \frac{1}{\pi} \int_{D(O ; 1)} f_{T}^{*}\left(\cdot, S_{2}, S_{3}\right) S_{3} d S_{2} d S_{3}+\frac{1}{\pi} \int_{0}^{2 \pi} g_{T}^{*}(\cdot, \theta) \sin \theta d \theta \\
G= & G_{N} \vec{N}+G_{B} \vec{B},
\end{aligned}\right.
$$

where $f^{*}$ and $g^{*}$ were decomposed in the Frenet basis:

$$
\begin{aligned}
& f^{*}=f_{T}^{*} \cdot \vec{T}+f_{B}^{*} \cdot \vec{B}+f_{N}^{*} \cdot \vec{N}, \\
& g^{*}=g_{T}^{*} \cdot \vec{T}+g_{B}^{*} \cdot \vec{B}+g_{N}^{*} \cdot \vec{N} .
\end{aligned}
$$

The following result holds:

Proposition 6.1. Let $u^{\delta}$ be the solution of problem (6.1) with $F^{\delta}$ and $G^{\delta}$ satisfying (6.6). Assume furthemore, that

$$
\int_{0}^{L} F_{E} \cdot V d s=0, \quad \forall V \in D_{\text {In }} .
$$

Then, $u^{\delta}$ satisfies the a priori estimate

$$
\left|u^{\delta}\right|_{\mathcal{E}} \leq C \delta
$$

REMARK 6.2. Hypothesis (6.8) means that $F_{E}$ does not have any contribution in the inextensional displacements.

Proof of Proposition 6.1. From Section 4, we know that $u^{\delta}$ can be written in the form (see (5.1)),

$$
\begin{aligned}
u^{\delta}\left(s, s_{2}, s_{3}\right)= & U_{I}^{\delta}(s)+U_{E}^{\delta}(s)-\left(s_{2} \frac{d U_{I}^{\delta}}{d s}(s) \cdot \vec{N}(s)+s_{3} \frac{d U_{I}^{\delta}}{d s}(s) \cdot \vec{B}(s)\right) \vec{T}(s) \\
& +\left(-s_{3} \vec{N}(s)+s_{2} \vec{B}(s)\right) \Theta_{u}^{\delta}(s)+\delta \widehat{u}^{\delta}\left(s, \frac{s_{2}}{\delta}, \frac{s_{3}}{\delta}\right), \quad \forall\left(s, s_{2}, s_{3}\right) \in \omega_{\delta},
\end{aligned}
$$

with $U_{I}^{\delta} \in D_{I n}, U_{E}^{\delta} \in D_{E x}$ and $\widehat{u}^{\delta}$ is defined by (5.2). Let choose $u^{\delta}$ as test function in (6.2). Recalling notation (6.7), hypothesis (6.8) implies that for the right-hand side term we have

$$
\left\{\begin{aligned}
\int_{\omega_{\delta}} F^{\delta} \cdot u^{\delta} & d \omega_{\delta}+\int_{\partial \omega_{\delta}} G^{\delta} \cdot u^{\delta} d_{s} \omega_{\delta}=\int_{\omega_{\delta}} F^{\delta} \cdot \delta \widehat{u}^{\delta} d \omega_{\delta}+\int_{\partial \omega_{\delta}} G^{\delta} \cdot \delta \widehat{u}^{\delta} d_{s} \omega_{\delta} \\
& +\pi \delta^{2} \int_{0}^{L}\left[F_{E} \cdot U_{E}^{\delta}+\delta F_{I} \cdot\left(U_{E}^{\delta}+U_{I}^{\delta}\right)-\delta G \cdot \frac{d U_{I}^{\delta}}{d s}+\delta F_{T} \Theta^{\delta}\right] d s .
\end{aligned}\right.
$$


Making use in (6.10) of estimte (5.3), and recalling the ellipticity condition (6.2), we have

$$
C_{0}\left|u^{\delta}\right|_{\mathcal{E}}^{2} \leq \int_{\mathcal{P}_{\delta}} a_{i j k h} \gamma_{i j}\left(u^{\delta}\right) \gamma_{k h}\left(u^{\delta}\right) d x=\int_{\mathcal{P}_{\delta}} F^{\delta} \cdot u^{\delta} d x+\int_{\Gamma_{\delta}} G^{\delta} \cdot u^{\delta} d_{s} x \leq C \delta\left|u^{\delta}\right|_{\mathcal{E}}
$$

whence the a priori estimate (6.9) is straightforward.

Corollary 6.3. For the sequence $\left(u^{\delta}\right)$ one has convergences (5.4), (5.7) and (5.8) (where one has to replace $w$ by $u$ and $W$ by $U)$. Moreover, for $\delta \rightarrow 0$,

$$
u^{\delta}\left(s, s_{2}, s_{3}\right) \sim \frac{1}{\delta} U_{I}(s)+U^{\star}\left(s, \frac{s_{2}}{\delta}, \frac{s_{3}}{\delta}\right)+\delta \widehat{u}\left(s, \frac{s_{2}}{\delta}, \frac{s_{3}}{\delta}\right), \quad \forall\left(s, s_{2}, s_{3}\right) \in \omega_{\delta},
$$

with $U_{I}, U^{\star}$ and $\widehat{u}$ given by Proposition 5.1.

Proof. The result is a simple consequence of the fact that a priori estimate (6.9) is precisely hypothesis (5.3) from Propositions 5.1 and 5.2.

Proposition 5.1 gives, besides $U_{I}, U^{\star}$ and $\widehat{u}$, also the limit functions $U_{E}, \mathcal{R}_{u}^{\star}$ and $\Theta_{u}^{\star}=\mathcal{R}_{u}^{\star} \cdot \vec{T}$. In the next section, we will show that the pair $\left(U_{I}, \Theta_{u}^{\star}\right)$ is the solution of a system of fourth order partial differential equations, while $U_{E}$ is the solution of a system of second order ones. This will completely determine all the limit functions mentioned above.

\section{Asymptotic behaviour of curved rods.}

We start this section by studying the behaviour of the limit residual displacement $\widehat{u}^{\delta}$. To this end, let $v$ be such that

$$
v\left(s, s_{2}, s_{3}\right)=\delta \psi\left(s, \frac{s_{2}}{\delta}, \frac{s_{3}}{\delta}\right), \quad \text { where } \psi \in H^{1}\left(\omega ; \mathbb{R}^{3}\right) \cap \mathcal{V}_{m}, \quad \text { with } \psi=0 \quad \text { on } \quad \Gamma_{0} .
$$

Then, an straightforward computation leads to the expressions

$$
\begin{aligned}
& e_{11}\left(v^{\delta}\right)=\frac{\delta}{1-\delta c S_{2}}\left[\frac{\partial \psi}{\partial s} \cdot \vec{T}+\tau S_{3}\left(\frac{\partial \psi}{\partial S_{2}} \cdot \vec{T}\right)-\tau S_{2}\left(\frac{\partial \psi}{\partial S_{3}} \cdot \vec{T}\right)\right], \\
& e_{12}\left(v^{\delta}\right)=\frac{1}{2}\left(\frac{\partial \psi}{\partial S_{2}} \cdot \vec{T}\right)+\frac{\delta}{2}\left(\frac{\partial \psi}{\partial s} \cdot \vec{N}\right)+\frac{\delta}{2\left(1-\delta c S_{2}\right)}\left(c S_{2} \frac{\partial \psi}{\partial s}+\tau S_{3} \frac{\partial \psi}{\partial S_{2}}-\tau S_{2} \frac{\partial \psi}{\partial S_{3}}\right) \cdot \vec{N} \\
& e_{13}\left(v^{\delta}\right)=\frac{1}{2}\left(\frac{\partial \psi}{\partial S_{3}} \cdot \vec{T}\right)+\frac{\delta}{2}\left(\frac{\partial \psi}{\partial s} \cdot \vec{B}\right)+\frac{\delta}{2\left(1-\delta c S_{2}\right)}\left(c S_{2} \frac{\partial \psi}{\partial s}+\tau S_{3} \frac{\partial \psi}{\partial S_{2}}-\tau S_{2} \frac{\partial \psi}{\partial S_{3}}\right) \cdot \vec{B},
\end{aligned}
$$

which immediately imply the following strong convergences in $L^{2}(\omega)$ :

$$
\mathcal{T}_{\delta}\left(e_{11}\left(v^{\delta}\right)\right) \longrightarrow 0, \quad \mathcal{T}_{\delta}\left(e_{12}\left(v^{\delta}\right)\right) \longrightarrow \frac{1}{2} \frac{\partial \psi}{\partial S_{2}} \cdot \vec{T}, \quad \mathcal{T}_{\delta}\left(e_{13}\left(v^{\delta}\right)\right) \longrightarrow \frac{1}{2} \frac{\partial \psi}{\partial S_{3}} \cdot \vec{T}
$$

Moreover, $e_{22}, e_{33}$ and $e_{23}$ are independent of $\delta$, since

$$
e_{22}\left(v^{\delta}\right)=\frac{\partial \psi}{\partial S_{2}} \cdot \vec{N}, \quad e_{33}\left(v^{\delta}\right)=\frac{\partial \psi}{\partial S_{3}} \cdot \vec{B}, \quad e_{23}\left(v^{\delta}\right)=\frac{1}{2}\left(\frac{\partial \psi}{\partial S_{3}} \cdot \vec{N}+\frac{\partial \psi}{\partial S_{2}} \cdot \vec{B}\right)
$$


Let now take $v^{\delta}$ defined by (7.1) as test displacement in (6.3) where we pass to the limit as $\delta \rightarrow 0$ by using Corollary 6.3 and (7.2). Taking into account (7.3), we obtain

$$
\left\{\begin{array}{l}
\mu \int_{\omega}\left[-c S_{3}\left(\frac{d U_{I}}{d s} \cdot \vec{B}\right)-S_{3} \frac{d \Theta_{u}^{\star}}{d s}-\tau S_{2} \Theta_{u}^{\star}+\frac{\partial \widehat{u}}{\partial S_{2}} \cdot \vec{T}\right]\left(\frac{\partial \psi}{\partial S_{2}} \cdot \vec{T}\right) d \omega \\
\quad+\mu \int_{\omega}\left[c S_{2}\left(\frac{d U_{I}}{d s} \cdot \vec{B}\right)+S_{2} \frac{d \Theta_{u}^{\star}}{d s}+\tau S_{3} \Theta_{u}^{\star}+\frac{\partial \widehat{u}}{\partial S_{3}} \cdot \vec{T}\right]\left(\frac{\partial \psi}{\partial S_{3}} \cdot \vec{T}\right) d \omega \\
\quad+\int_{\omega} \lambda\left[\frac{d U_{E}}{d s} \cdot \vec{T}-S_{2}\left(\frac{d^{2} U_{I}}{d s^{2}} \cdot \vec{N}\right)-S_{3}\left(\frac{d^{2} U_{I}}{d s^{2}} \cdot \vec{B}\right)+c S_{3} \Theta_{u}^{\star}\right]\left(\frac{\partial \psi}{\partial S_{2}} \cdot \vec{N}\right) d \omega \\
\quad+\int_{\omega}\left[(\lambda+2 \mu)\left(\frac{\partial \widehat{u}}{\partial S_{2}} \cdot \vec{N}\right)+\lambda\left(\frac{\partial \widehat{u}}{\partial S_{3}} \cdot \vec{B}\right)\right]\left(\frac{\partial \psi}{\partial S_{2}} \cdot \vec{N}\right) d \omega \\
\quad+\int_{\omega} \lambda\left[\frac{d U_{E}}{d s} \cdot \vec{T}-S_{2}\left(\frac{d^{2} U_{I}}{d s^{2}} \cdot \vec{N}\right)-S_{3}\left(\frac{d^{2} U_{I}}{d s^{2}} \cdot \vec{B}\right)+c S_{3} \Theta_{u}^{\star}\right]\left(\frac{\partial \psi}{\partial S_{3}} \cdot \vec{B}\right) d \omega \\
\quad+\int_{\omega}\left[\lambda\left(\frac{\partial \widehat{u}}{\partial S_{2}} \cdot \vec{N}\right)+(\lambda+2 \mu)\left(\frac{\partial \widehat{u}}{\partial S_{3}} \cdot \vec{B}\right)\right]\left(\frac{\partial \psi}{\partial S_{3}} \cdot \vec{B}\right) d \omega \\
\quad+\mu \int_{\omega}\left(\frac{\partial \widehat{u}}{\partial S_{3}} \cdot \vec{N}+\frac{\partial \widehat{u}}{\partial S_{2}} \cdot \vec{B}\right)\left(\frac{\partial \psi}{\partial S_{3}} \cdot \vec{N}+\frac{\partial \psi}{\partial S_{2}} \cdot \vec{B}\right) d \omega=0 .
\end{array}\right.
$$

Since $H^{1}\left(\omega ; \mathbb{R}^{3}\right)$ is dense in $H_{\mathbb{R}^{3}}\left(\left\{\partial_{S_{2}}, \partial_{S_{3}}\right\} ; \omega\right),(7.4)$ holds for any $\psi \in H_{\mathbb{R}^{3}}\left(\left\{\partial_{S_{2}}, \partial_{S_{3}}\right\} ; \omega\right)$.

Now, observe that (7.4) decouples. On one hand, for any $\chi \in H_{\mathbb{R}}\left(\left\{\partial_{S_{2}}, \partial_{S_{3}}\right\} ; \omega\right)$ one has

$$
\int_{\omega}\left(-\tau S_{2} \Theta_{u}^{\star}+\frac{\partial \widehat{u}}{\partial S_{2}} \cdot \vec{T}\right) \frac{\partial \chi}{\partial S_{2}} d x+\int_{\omega}\left(\tau S_{3} \Theta_{u}^{\star}+\frac{\partial \widehat{u}}{\partial S_{3}} \cdot \vec{T}\right) \frac{\partial \chi}{\partial S_{3}} d x=0 .
$$

On the other hand, if $\left(\psi_{2}, \psi_{3}\right) \in H_{\mathbb{R}^{2}}\left(\left\{\partial_{S_{2}}, \partial_{S_{3}}\right\} ; \omega\right)$, then

$$
\begin{aligned}
\int_{\omega}\left[(\lambda+2 \mu)\left(\frac{\partial \widehat{u}}{\partial S_{2}} \cdot \vec{N}\right)\right. & \left.+\lambda\left(\frac{\partial \widehat{u}}{\partial S_{3}} \cdot \vec{B}\right)\right] \frac{\partial \psi_{2}}{\partial S_{2}} d \omega \\
& +\int_{\omega}\left[\lambda\left(\frac{\partial \widehat{u}}{\partial S_{2}} \cdot \vec{N}\right)+(\lambda+2 \mu)\left(\frac{\partial \widehat{u}}{\partial S_{3}} \cdot \vec{B}\right)\right] \frac{\partial \psi_{3}}{\partial S_{3}} d \omega \\
& +\mu \int_{\omega}\left(\frac{\partial \widehat{u}}{\partial S_{3}} \cdot \vec{N}+\frac{\partial \widehat{u}}{\partial S_{2}} \cdot \vec{B}\right)\left(\frac{\partial \psi_{2}}{\partial S_{3}}+\frac{\partial \psi_{3}}{\partial S_{2}}\right) d \omega \\
= & -\lambda \int_{\omega}\left[\frac{d U_{E}}{d s} \cdot \vec{T}-S_{2}\left(\frac{d^{2} U_{I}}{d s^{2}} \cdot \vec{N}\right)-S_{3}\left(\frac{d^{2} U_{I}}{d s^{2}} \cdot \vec{B}\right)\right. \\
& \left.+c S_{3} \Theta_{u}^{\star}\right]\left(\frac{\partial \psi_{2}}{\partial S_{2}}+\frac{\partial \psi_{3}}{\partial S_{3}}\right) d \omega .
\end{aligned}
$$

This problem, as well as (7.5) can be solved explicitly (see Griso [5]), to get the limit residual displacement $\widehat{u}$ expressed in terms of extensional and inextensional displacements, and of the torsion angle of the rod,

$$
\begin{aligned}
& \widehat{u} \cdot \vec{T}=\frac{S_{2}^{2}-S_{3}^{2}}{2} \tau \Theta_{u}^{\star}, \\
& \widehat{u} \cdot \vec{N}=\nu\left[-S_{2} \frac{d U_{E}}{d s}+\frac{S_{2}^{2}-S_{3}^{2}}{2}\left(\frac{d^{2} U_{I}}{d s^{2}} \cdot \vec{N}\right)+S_{2} S_{3}\left(-c \Theta_{u}^{\star}+\frac{d^{2} U_{I}}{d s^{2}} \cdot \vec{B}\right)\right], \\
& \widehat{u} \cdot \vec{B}=\nu\left[-S_{3} \frac{d U_{E}}{d s}+S_{2} S_{3}\left(\frac{d^{2} U_{I}}{d s^{2}} \cdot \vec{N}\right)+\frac{S_{3}^{2}-S_{2}^{2}}{2}\left(-c \Theta_{u}^{\star}+\frac{d^{2} U_{I}}{d s^{2}} \cdot \vec{B}\right)\right],
\end{aligned}
$$

where $\nu$ is the Poisson coefficient

$$
\nu=\frac{\lambda}{2(\lambda+\nu)} .
$$


We are also able to give the limit in $L^{2}\left(\omega ; \mathbb{R}^{9}\right)$ of the stress tensor defined by (6.4). Indeed, by using convergences (5.8) one easily has

$$
\mathcal{T}_{\delta}\left(\sigma_{i j}\left(u^{\delta}\right)\right) \rightarrow \sigma_{i j} \quad \text { weakly in } L^{2}(\omega)
$$

where

$$
\begin{aligned}
& \sigma_{12}=\sigma_{21}=-\frac{\mu S_{3}}{2}\left[c\left(\frac{d U_{I}}{d s} \cdot \vec{B}\right)+\frac{d \Theta_{u}^{*}}{d s}\right], \\
& \sigma_{13}=\sigma_{31}=\frac{\mu S_{2}}{2}\left[c\left(\frac{d U_{I}}{d s} \cdot \vec{B}\right)+\frac{d \Theta_{u}^{*}}{d s}\right], \\
& \sigma_{22}=\sigma_{33}=\sigma_{23}=\sigma_{32}=0 .
\end{aligned}
$$

A simple computation gives

$$
\begin{aligned}
& \sigma_{22}=\lambda e_{11}+(\lambda+2 \mu) e_{22}+\lambda e_{33}=0 \\
& \sigma_{33}=\lambda e_{11}+\lambda e_{22}+(\lambda+2 \mu) e_{33}=0 .
\end{aligned}
$$

Consequently, $e_{22}=-\nu e_{11}$ and $e_{33}=-\nu e_{11}$. Hence, one can give the expression of $\sigma_{11}$ in terms of $e_{11}$,

$$
\sigma_{11}=(\lambda+2 \mu) e_{11}+\lambda e_{22}+\lambda e_{33}=E e_{11}=E\left[\frac{d U_{E}}{d s} \cdot \vec{T}-S_{2}\left(\frac{d^{2} U_{I}}{d s^{2}} \cdot \vec{N}\right)-S_{3}\left(\frac{d^{2} U_{I}}{d s^{2}} \cdot \vec{B}-c \Theta_{u}^{\star}\right)\right]
$$

where $E$ is the Young modulus of the material from which the rod is made,

$$
E=\frac{\mu(3 \lambda+2 \mu)}{\lambda+\mu}
$$

Collecting all the above information, we now can state the main theorems giving the asymptotic behaviour of the curved $\operatorname{rod} \mathcal{P}_{\delta}$.

Theorem 7.1. (Asymptotic behaviour of the inextensional displacement and of the torsion angle). The couple $\left(U_{I}, \Theta_{u}^{*}\right) \in \mathcal{D}_{\text {In }} \times \mathcal{V}_{1}$, is the solution of the variational problem

$$
\left\{\begin{aligned}
\frac{E}{3} \int_{0}^{L}\left[\left(\frac{d^{2} U_{I}}{d s^{2}} \cdot \vec{N}\right)\right. & \left.\left(\frac{d^{2} V}{d s^{2}} \cdot \vec{N}\right)+\left(-c \Theta_{u}^{*}+\frac{d^{2} U_{I}}{d s^{2}} \cdot \vec{B}\right)\left(-c \psi+\frac{d^{2} V}{d s^{2}} \cdot \vec{B}\right)\right] d s \\
& +\frac{\mu}{3} \int_{0}^{L}\left(\frac{d \Theta_{u}^{*}}{d s}+c \frac{d U_{I}}{d s} \cdot \vec{B}\right)\left(\frac{d \psi}{d s}+c \frac{d V}{d s} \cdot \vec{B}\right) d s \\
= & \int_{0}^{L}\left(F_{I} \cdot V-G \cdot \frac{d V}{d s}+F_{T} \psi\right) d s, \quad \forall(V, \psi) \in \mathcal{D}_{\text {In }} \times \mathcal{V}_{1},
\end{aligned}\right.
$$

where $\mathcal{V}_{1}=\left\{\psi \in H^{1}(0, L) \mid \psi(0)=0\right\}$

Theorem 7.2. (Asymptotic behaviour of the extensional displacement). The limit extensional displacement $U_{E} \in D_{E x}$ is the solution of the variational problem

$$
E \int_{0}^{L} \frac{d U_{E}}{d s} \cdot \frac{d V}{d s} d s=\int_{0}^{L} F_{E} \cdot V d s, \quad \forall V \in D_{E x}
$$

Proof of Theorems 7.1 and 7.2. Let $V \in \mathcal{D}_{\text {In }}$ and $\psi \in \mathcal{V}_{1}$ and take in (6.3) the test-displacement $v^{\delta} \in H_{\Gamma_{0}}^{1}\left(\mathcal{P}_{\delta} ; \mathbb{R}^{3}\right)$, defined for any $s \in \omega_{\delta}$ by

$$
v^{\delta}(s)=\frac{1}{\delta} V(s)-\left[\frac{s_{2}}{\delta}\left(\frac{d V}{d s}(s) \cdot \vec{N}(s)\right)+\frac{s_{3}}{\delta}\left(\frac{d V}{d s}(s) \cdot \vec{B}(s)\right)\right] \vec{T}(s)+\left[-\frac{s_{3}}{\delta} \vec{N}(s)+\frac{s_{2}}{\delta} \vec{B}(s)\right] \psi(s)
$$


With this choice, the components of the deformation tensor $e_{i j}\left(v^{\delta}\right)$ converge strongly in $L^{2}(\omega)$ as follows:

$$
\begin{aligned}
& \mathcal{T}_{\delta}\left(e_{11}\left(v^{\delta}\right)\right) \longrightarrow-S_{2}\left(\frac{d^{2} V}{d s^{2}} \cdot \vec{N}\right)-S_{3}\left(\frac{d^{2} V}{d s^{2}} \cdot \vec{B}\right)+c S_{3} \psi, \\
& \mathcal{T}_{\delta}\left(e_{12}\left(v^{\delta}\right)\right) \longrightarrow \frac{1}{2}\left[-c S_{3}\left(\frac{d V}{d s} \cdot \vec{B}\right)-S_{3} \frac{d \psi}{d s}\right] \\
& \mathcal{T}_{\delta}\left(e_{13}\left(v^{\delta}\right)\right) \longrightarrow \frac{1}{2}\left[c S_{2}\left(\frac{d V}{d s} \cdot \vec{B}\right)+S_{2} \frac{d \psi}{d s}\right], \\
& \mathcal{T}_{\delta}\left(e_{22}\left(v^{\delta}\right)\right)=0, \quad \mathcal{T}_{\delta}\left(e_{33}\left(v^{\delta}\right)\right)=0, \quad \mathcal{T}_{\delta}\left(e_{23}\left(v^{\delta}\right)\right)=0 .
\end{aligned}
$$

Making use of these convergences when passing to the limit in problem (6.3), yields (7.6).

Let now $V \in D_{E x}$. Since $V$ is an admissible displacement of the rod (see (3.11)), it can be taken as test-displacement in (6.3.). Passing to the limit and using Corollary 6.3, (7.7) follows easily.

REMARK 7.3. The variational problems (7.6) and (7.7) are coercive. Hence the whole sequences in (5.4) and (5.8) converge.

REmark 7.4. Convergences in (5.4.) and (5.9.) are strong. Indeed, we have

$$
\begin{aligned}
\frac{1}{\pi} \int_{\omega} a_{i j k l} e_{i j} e_{k l} d \omega & \leq \lim _{\delta \rightarrow 0} \frac{1}{\pi \delta^{2}} \int_{\omega_{\delta}} a_{i j k l} e_{i j}\left(u^{\delta}\right) e_{k l}\left(u^{\delta}\right) d \omega_{\delta} \\
& \leq \varlimsup_{\delta \rightarrow 0} \frac{1}{\pi \delta^{2}} \int_{\omega_{\delta}} a_{i j k l} e_{i j}\left(u^{\delta}\right) e_{k l}\left(u^{\delta}\right) d \omega_{\delta} \\
& =\varlimsup_{\delta \rightarrow 0} \frac{1}{\pi \delta^{2}}\left[\int_{\omega} F^{\delta} \cdot u^{\delta} d \omega+\int_{\partial \omega} G^{\delta} \cdot u^{\delta} d_{s} \omega\right] \\
& =\int_{0}^{L}\left[F_{E} \cdot U_{E}+F_{I} \cdot U_{I}-G \cdot \frac{d U_{I}}{d s}+F_{T} \cdot \Theta_{u}^{*}\right] d s \\
& =\frac{1}{\pi} \int_{\omega} a_{i j k l} e_{i j} e_{k l} d \omega
\end{aligned}
$$

\section{Final comments and remarks.}

Theorem 3.3 has been proved in the particular case of a curved rod whose cross section is a disc. This theorem still holds when the rod cross section is a connected bounded open set with a Lipschitz boundary. It also holds when the rod cross section is not fixed but depends on the heights where it is taken.

The residual displacement introduced in Section 4 (Definition 4.2) takes into account the behaviour of the cross sections of the rod. For the sake of simplicity, to explain the action of the limit residual displacements, we restricted ourselves to the case of a rod whose cross section is a disc, the applied forces concerning only the rod elementary displacements. There is no difficulty to extend this explanation to more general cases.

We have obtained, after passing to the limit, two uncoupled limit problems, the first one linking the inextensional displacement and the rod torsion angle, the second one defining the extensional displacement. Such an uncoupling is due to the smoothness of the rod on one hand, and on the other hand, to the symmetries of the cross-section. In a more general case, where either the rod has a variable cross-section, or it is made by a non homogeneous or an anisotropic material, we obtain only one limit problem linking together the extensional displacement, the inextensional one and the torsion angle.

Obvious modifications of the functional spaces introduced above (see (4.1), (4.2)), will give the asymptotic behaviour of curved rods clamped at both ends or of closed curved rods. For example, in the case of a 
curved rod clamped at both ends, the set of inextensional displacements will be defined by

$$
D_{\text {In }}=\left\{U \in H_{0}^{1}\left(0, L ; \mathbb{R}^{3}\right) \mid \frac{d U}{d s} \cdot \vec{T}=0\right\} .
$$

The space $H_{0}^{1}\left(0, L ; \mathbb{R}^{3}\right)$ is equipped with inner product

$$
\langle U, V\rangle=\int_{0}^{L} \frac{d U}{d s} \cdot \frac{d V}{d s} d s .
$$

The extensional displacements space is then, by definition, the orthogonal space of $D_{\text {In }}$ in $H_{0}^{1}\left(0, L ; \mathbb{R}^{3}\right)$ for the inner product $\langle\cdot, \cdot\rangle$. Proceeding as we have done above, we obtain the same problems (7.6) and (7.7) provided the functional spaces are changed in order to take into account the fact that the rod is clamped at the both ends.

In the case of straight rods, the curvature $c$ and torsion $\tau$ vanish. All the results are obviously true and we obtain the asymptotic behaviour of straight rods.

\section{Acknowledgements}

The author wishes to thank Doina Cioranescu for her unfailing support and her helpfulness throughout the writing of this paper.

\section{REFERENCES}

[1] K. Arunakirinathar and B. D. Reddy, Mixed finite element methods for elastic rods of arbitrary geometry. Numer. Math., 1, 64 (1993), 13-43.

[2] D. Cioranescu, O. A. Oleinik and G. Tronel, Korn's inequalities for frame type structures and junctions with sharp estimates for the constants. Asymptotic Analysis, 8 (1994), 1-14.

[3] D. Cioranescu, A. Damlamian and G. Griso, Periodic unfolding and homogenization, C. R. Acad. Sci. Paris, Ser. I 335 (2002), 99-104.

[4] G. Griso, Etude asymptotiques de structures réticulées minces. Thèse Université Pierre et Marie Curie (Paris VI), 1995.

[5] G. Griso, Asymptotic behavior of structures made of plates. C. R. Acad. Sci. Paris, Ser. I 336 (2003), 101-106.

[6] R. Jamal and E. Sanchez-Palencia, Théorie asymptotique des tiges courbes anisotropes, C. R. Acad. Sci. Paris, Ser. I 332 (1996), 1099-1106.

[7] M. Jurak and J. Tambača, Derivation and justification of a curved rod model. Math. Models Methods Appl. Sci. 9, 7 (1999), 991-1014.

[8] M. Jurak and J. Tambača, Linear curved rod model. General curve. Math. Models Methods Appl. Sci. 11, 7 (2001), 1237-1252.

[9] A. Kondratiev and O .A. Oleinik, On Korn's inequalities. C. R. Acad. Sci. Paris, Ser. I 308 (1989), 483-487.

[10] H. Le Dret, Modeling of the junction between two rods, J. Math. Pures Appl. 68 (1989), 365-397.

[11] J. Lelong-Ferrand and J.M. Arnaudiès, Cours de mathématiques, Tome 3, Géométrie et cinématique. Dunod Université (1979).

[12] F. Murat and A. Sili, Comportement asymptotique des solutions du système de l'élasticité linéarisée anisotrope hétérogène dans des cylindres minces. C. R. Acad. Sci. Paris, Ser. I 328 (1999), 179-184.

[13] F. Murat and A. Sili, Effets non locaux dans le passage 3d-1d en élasticité linéarisée anisitrope hétérogène. C. R. Acad. Sci. Paris, Ser. I 330 (2000), 745-750. 
[14] S. Neunkirch, G.H. Vander Heijden and J.M. Thompson, Writhing, instabilities of twisted rods; from infinite to finite length. J. Math. Phys. Solids 50, 6 (2002), 1175-1191.

[15] J. Sanchez-Hubert and E. Sanchez-Palencia, Statics of curved rods on account of torsion and flexion. European Jour. Mechanics A/Solids, 18 (1999), 365-390.

[16] E. Sanchez-Palencia, Sur certains problèmes de couplage de plaques et de barres. Equations aux dérivées partielles et applications. Articles dédiés à J.L. Lions, Gauthier-Villars (1998), 725-744.

[17] J. Tambača. Justification of the dynamic model of curved rods, Asymptot. Anal. 31, 1 (2002), 43-68.

[18] L. Trabucho and J. M. Viano, Mathematical modeling of rods, in Handbook of Numerical Analysis. 487-974, ed. P.G. Ciarlet and J.L. Lions, North-Holland, Amsterdam, 1996. 